\title{
A Tutorial for Olfaction-based Multisensorial Media Application Design and Evaluation
}

\author{
NIALL MURRAY, Athlone Institute of Technology, Ireland \\ OLUWAKEMI A. ADEMOYE, University of Wales, UK \\ GHEORGHITA GHINEA, Brunel University, UK \\ GABRIEL-MIRO MUNTEAN, Dublin City University, Ireland
}

\begin{abstract}
Recently, multimedia researchers have added several so called new media to the traditional multimedia components (e.g. olfaction, haptic and gustation). Evaluating multimedia user perceived Quality of Experience (QoE) is already non-trivial and the addition of multisensorial media components increases this challenge. No standardized methodology exists to conduct subjective quality assessments of multisensorial media applications. To date researchers have employed different aspects of audiovisual standards to assess user QoE of multisensorial media applications and thus, a fragmented approach exists. In this paper, the authors highlight issues researchers face from numerous perspectives including applicability (or lack of) existing audiovisual standards to evaluate user QoE and lack of result comparability due to varying approaches, specific requirements of olfactory-based multisensorial media applications, and novelty associated with these applications. Finally, based on the diverse approaches in the literature and the collective experience of authors, this paper provides a tutorial and recommendations on the key steps to conduct olfactory-based multisensorial media QoE evaluation.
\end{abstract}

- Information system $\rightarrow$ Database management system $\rightarrow$ Information systems applications $\rightarrow$ Multimedia information systems.

Additional Key Words and Phrases: Quality of Experience, multisensorial media, olfaction, evaluation

ACM Reference Format:

Niall Murray, Oluwakemi A. Ademoye, Gheorghita Ghinea, and Gabriel-Miro Muntean, 2016. A Tutorial for Olfaction-based Multisensorial Media Application Design and Evaluation. ACM Computer Surveys, 2016

\section{INTRODUCTION}

A brief perusal of multimedia research published in the last decade shows a significant increase in the number multimedia applications incorporating media components outside the traditional audio and video. Such works include olfaction (sense of smell) [1], haptic (sense of touch) [2] and, to a lesser extent, gustation (sense of taste) [3]. These types of experiences have been reflected by different terms over the years including multimodal media [41], sensory experiences [27], multisensory experiences [87], multiple sensorial media (mulsemedia) and multisensorial media [4][5]. For consistency in the remainder of this paper, the term mulsemedia is proposed to reflect the use of sensory components in our research and related works.

Authors' addresses: N. Murray, Department of Electronics and Informatics and the Software Research Institute, Athlone Institute of Technology, Ireland (e-mail: nmurray@research.ait.ie), O. A. Ademoye, School of Applied Computing, University of Wales, Trinity Saint David, Wales (e-mail: kemi.ademoye@sm.uwtsd.ac.uk), G. Ghinea, Department of Computer Science, Brunel University, UK, (email: george.ghinea@brunel.ac.uk), G.-M. Muntean, School of Electronic Engineering, Dublin City University, Ireland (e-mail: gabriel.muntean@dcu.ie).

"Permission to make digital or hardcopies of part or all of this work for personal or classroom use is granted without fee provided that copies are not made or distributed for profit or commercial advantage and that copies show this notice on the first page or initial screen of a display along with the full citation. Copyrights for components of this work owned by others than ACM must be honored. Abstracting with credits permitted. To copy otherwise, to republish, to post on servers, to redistribute to lists, or to use any component of this work in other works requires prior specific permission and/or a fee. Permissions may be requested from Publications Dept., ACM, Inc., 2 Penn Plaza, Suite 701, New York, NY 10121-0701 USA, fax +1 (212) 869-0481, or permissions@acm.org."

() 2016 ACM 1539-9087/2010/03-ART39 $\$ 15.00$

DOI: http://dx.doi.org/10.1145/0000000.0000000

ACM Computer Surveys, Vol., No., Article, Publication date: 
The motivation for including diverse sensorial media components has generally been to increase the level of user immersion and/or Quality of Experience (QoE), which refers to the "degree of delight or annoyance of applications or service" [6]. It considers the influence of communication services, application type, network, device, context of use, content, user personality, etc. on user QoE [6].

A key work encouraging and outlining potential future research directions in the area of multimedia communication was published by Rowe and Jain [7]. This paper was the result of discussions between thirty leading researchers from the multimedia research domain. Three key themes were identified by this group: (a) multimedia systems or applications stimulate more than one sense in a correlated manner (b) multimedia systems should be integrated and be adaptable to varying network conditions and user perception (c) multimedia systems can be multimodal and interactive. It is valid to suggest that the emergence of mulsemedia as a research field has evolved from each of these themes. Theme (a) is supported as a mulsemedia application is defined which stimulates three or more senses. With respect to theme (b), the delivery of mulsemedia metadata can be adapted based on network conditions (as outlined in [8]), but also based on user preferences and perception. Finally, theme (c) is supported by the very nature of mulsemedia i. e. it stimulates multiple human senses and supports user interaction. It was not the aim of these researchers to highlight approaches to evaluate multimedia quality, but the paper did infer that heuristic criteria were seen as key to any quality evaluation.

Several surveys have presented and discussed solutions to support QoS and QoEoriented multimedia communications. Seufert et al. [9] provided a survey of approaches for enhancing user QoE by employing video adaptation which considers on one hand user characteristics and on the other network conditions. In terms of the former research avenue, Nunes et al. [10] surveyed works that consider psychological states, human intents, emotions and actions inferred from sensory data in the human-system interaction process. In terms of the latter research direction, Juluri et al. [11] presented a tutorial on video streaming techniques and discussed various metrics for objective quantification of QoE of video streaming. They also surveyed apparatus and measurement approaches used to predict the user quality of experience in the context of video streaming. Seeling and Reisslein [12] performed a detailed evaluation of video transport mechanisms and their associated QoS in the context of H.264 video delivery. Kennedy et al., [13] discussed approaches for achieving balance between QoS/QoE and energy consumption during multimedia delivery. Finally, Chen et al. [14] presented a comprehensive review of video quality assessment methodologies with respect to analysis of video quality and relationship between QoS and QoE, highlighting also several potential future directions for QoE research. However none of these surveys have focused on multisensorial media communications and in particular on user QoE evaluation in multisensorial context.

Recently, individual articles have highlighted opportunities for mulsemedia research in general and for olfaction-related studies in particular. In [15], several olfaction-based mulsemedia applications were discussed based on a study that captured feedback via an online study on participant experience with olfaction. The authors defined the following categories: associating smell with the past; 
remembering through smell; stimulation of smell with experiences; scent creating a desire for more of a particular experience; identification through smell; power of smell; omnipresence of smell; effects of social interaction on smell; effects of olfaction on behavior or mood and finally expectations associated with smell. From an olfaction-based mulsemedia perspective, this complements two literature reviews: [16][17].

In [16], the use of olfaction-based mulsemedia in areas such as film, virtual reality, alerting systems, entertainment and gaming was presented. The influences of age, gender, culture, past experiences and emotion on perception of olfaction were discussed. The authors also highlighted a number of research directions for olfactionbased mulsemedia in terms of synchronization, content association and challenges with respect to olfactory display development. Another work by Murray et al., [17] complemented the former survey by presenting the application of the olfactory component in less apparent application domains such as health, tourism and education whilst classifying olfactory display development based on scent generation technique, application area, scent delivery capability and strengths/weaknesses of different approaches. It also highlighted research challenges with respect to QoE, the presentation of olfaction with other mulsemedia media components, and transmission of olfaction-based mulsemedia over constrained communication networks. Between these two works, which have focused on the use of olfaction as a media component, a comprehensive view of olfaction-based mulsemedia state of the art can be obtained. Another relevant article has focused solely on olfactory display design and development [18].

Whilst these works are valuable contributions, generally speaking, they have not considered the range of methodologies and approaches adopted to assess user QoE for olfaction-based mulsemedia. In this context, the authors have identified this as a valuable task that needs be addressed for olfaction-based mulsemedia systems. Generally the methodological approach outlined in the literature involved borrowing aspects of methodologies designed for traditional media components (e.g. audio or video). Such media components have generally been classified as being either discrete or continuous. However, it is debatable whether olfaction as a media component could be described as continuous or discrete media. Hence the authors question the applicability of these standards to mulsemedia and ask what additional measures are needed to accurately and consistently capture user QoE of olfaction-based mulsemedia?

The closest work in the literature to what the authors present in this paper is by Timmerer et al. [20]. They provided some recommendations in terms of existing ITU$\mathrm{T}$ standards [21][39] and their applicability for evaluations of sensory experiences. They also highlighted their own test design approach. Whilst it is a valuable and interesting article, no specific recommendations with respect to olfaction-based mulsemedia and its delivery were provided. Related to this, two works by Hamam et al. [22][23] proposed systems for evaluating QoE of haptic-based mulsemedia experiences. They applied a fuzzy logic system to the QoE modelling of haptic applications which considered traditional QoS metrics and human factors to quantitatively measure user QoE. 
This article is structured as follows: section 2 gives an outline of seven key research challenge areas that require efforts from the research community and section 3 provides an overview of the state of the art in the areas related to QoE assessment of olfaction-based mulsemedia. These works were compared and contrasted in terms of their methodologies, rating scales, sample sizes, sample balance, assessor screening and training, number and type of scents used and laboratory environment, inclusive of the methodologies employed for assessment. The authors own experiences from these perspectives of performing quality assessments of olfaction-based mulsemedia are also discussed. Finally, section 4 presents recommendations for mulsemedia quality evaluation in terms of laboratory design, assessor preparation, experimental design and unique characteristics of olfactionbased mulsemedia that should be considered. These recommendations are made based on the experience of the authors in the area of olfaction-based mulsemedia.

\section{RESEARCH CHALLENGES FOR OLFACTION-BASED MULTIMEDIA APPLICATIONS}

The emerging nature - in addition to the complexity of olfaction-based mulsemedia - results in a wide variety of research problems that needs to be considered. These range from re-evaluation of aspects that were previously executed in the audiovisual domain, with a focus on olfaction, to deeper understanding of how we perceive and consume olfaction based mulsemedia content, to application/domain specific research challenges for olfaction based mulsemedia applications.

\subsection{Olfaction based mulsemedia integration}

A key challenge for olfaction based mulsemedia applications is how we integrate the various modalities as a step towards truly immersive experiences and enhanced user QoE. It is salient to consider research going forward that focuses on recreating spatial and content relationships for olfaction based mulsemedia integration.

However, another interesting avenue is to consider solely what aspects the olfactory component can contribute, with a focus on what we wish to achieve by presenting the olfactory component. One example here is with respect to storytelling scenarios. Additional metadata in terms of what a story teller or director of an audiovisual content may want to evoke could theoretically be provided by an olfactory component, i.e. information not directly related to the audiovisual content could be supported via the presentation of an olfactory component.

\subsection{Synchronization}

Related to research challenge 2.1 , but more aligned with the traditional intermedia multimedia synchronization problem, is how we ensure the temporal relations between the various media component that reflect olfaction-based mulsemedia (audio, video, olfaction) are implemented. Each of these individual components has varying requirements from a temporal perspective. Although some studies exist with respect to olfaction-based mulsemedia synchronization, deeper analysis is required in terms of the influence of masking effects influence olfaction-based mulsemedia QoE.

An initial study on this topic is presented in [1], however further work is required based on context of audio and video media components and their influence on QoE. 


\subsection{Standardization}

The MPEG-V [19] standard ISO/IEC, of which there are 7 parts, "provides an architecture and specifies associated information representations to enable the interoperability between virtual worlds, as well as real and virtual worlds".

The standard entitled "Information technology — Media context and control" has the following parts:

- Architecture [19] - describes the overall MPEG-V architecture.

- Control Information [77] - describes the Control Information Description Language (CIDL) for controlling various sensory and display devices.

- Sensory Information [78] - introduces the "Sensory Effect Description Language (SEDL) and the Sensory Effect Vocabulary (SEV)" for describing sensory effects.

- Virtual World Object Characteristics [79] - introduces tools for describing virtual world objects' characteristics.

- Data formats for Interaction Devices [80] - presents the data format for exchanging information between diverse devices.

- Common Types and Tools [81] - describes common tools and data types used.

- Conformance and Reference Software [82] - introduces tools for generating and checking the conformance of MPEG-V descriptions.

Whilst the contribution of this standard is salient, more standardization efforts are required, in particular with respect to the olfactory component, but in particular with respect to the integration and synchronization aspects as highlighted in research challenges 2.1 and 2.2. It is salient to add, given the context of this paper, that we need a standardized methodological approach to context based QoE evaluation of mulsemedia applications.

\subsection{Olfactory sensor and display development}

The problems of how to capture, define metadata representations and present olfaction has proven a fundamental research challenge across a number of disciplines. Significant progress has been made with the recent development of more accurate sensors. This then facilitates the metadata modelling of the various chemical compounds. However, multimodal sensor ecosystems need to be developed. Only then, can real life representations of our natural world be presented as part of mulsemedia based systems. Of course, to achieve this, further development on the display side is required. Numerous commercial olfactory displays, as outlined in [16][17][83][105], are now available, but based on their design and implementation approaches, limitations exist in how we can control olfactory components in terms of intensity and duration.

Most scents come in liquid form or solids, such as gels and other porous materials, soaked with the scent. Thus, scent emission devices must vaporise their scents and transfer the scented air generated to the target, the human nose [18], [88]. Vaporisation of scents is achieved using four main techniques. Natural vaporisation requires no special mechanism, making this technique unsuitable for controlled scent emission. Accelerated air flow vaporisation is one of the popular techniques used for controlled scent emission and can be achieved via vaporising liquids such as essential oils from the surface, bubbling liquids or vaporising gels or porous materials. Heating of scented odorants and atomisation are the other two vaporisation techniques. 
Table I Summary of available olfactory displays

\begin{tabular}{|c|c|c|c|c|}
\hline Device & $\begin{array}{l}\text { Number of Scents } \\
\text { /Odorant Type }\end{array}$ & Availability & Device Control & $\begin{array}{l}\text { Method for } \\
\text { Scent } \\
\text { Vaporisation } \\
\text { + Delivery } \\
\end{array}$ \\
\hline 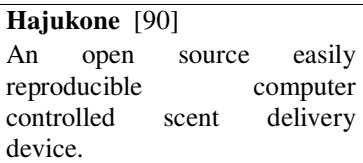 & $\begin{array}{l}\text { Capacity to emit six } \\
\text { scents. The scents } \\
\text { can be any liquid } \\
\text { based scent. }\end{array}$ & $\begin{array}{|lrr|}\text { Open } & \text { source } & \text { research } \\
\text { scent } & \text { controlled } \\
\text { device } & \text { that can be } \\
\text { built } & \text { with } & \text { low } \\
\text { technical skills. } & \end{array}$ & $\begin{array}{l}\text { API software to control } \\
\text { scent emission. }\end{array}$ & $\begin{array}{l}\text { Atomisation } \\
\text { combined } \\
\text { with air flow. }\end{array}$ \\
\hline $\begin{array}{l}\text { Simple Low-Cost Olfactory } \\
\text { Display USB Device [91] } \\
\text { A simple low-cost olfactory } \\
\text { display developed to address } \\
\text { the lack of adequate, } \\
\text { inexpensive devices to fulfil } \\
\text { research needs. }\end{array}$ & $\begin{array}{l}\text { One scent cartridge } \\
\begin{array}{l}\text { using a liquid } \\
\text { odorant. }\end{array}\end{array}$ & $\begin{array}{l}\text { Open source research } \\
\text { device roviding } \\
\text { detailed construction } \\
\text { details of both the } \\
\text { device and odorants } \\
\text { for others to replicate. }\end{array}$ & $\begin{array}{l}\text { API SDK software to } \\
\text { control scent emission } \\
\text { duration. }\end{array}$ & $\begin{array}{l}\text { Airflow } \\
\text { vaporisation } \\
\text { and delivery. }\end{array}$ \\
\hline $\begin{array}{l}\text { Digital Flavor Synthesizing } \\
\text { device [93] } \\
\text { A handheld, digital instrument } \\
\text { which combines the simulation } \\
\text { of taste and smell sensations to } \\
\text { create and simulate flavours. }\end{array}$ & $\begin{array}{l}\text { Capacity to store } \\
\text { and emit four } \\
\text { scents. Odorants are } \\
\text { solid perfume paste } \\
\text { gels. }\end{array}$ & Research device & $\begin{array}{l}\text { Pulse Width Modulation } \\
\text { (PWM) based technique }\end{array}$ & $\begin{array}{l}\text { Heating } \\
\text { vaporisation } \\
\text { combined } \\
\text { with airflow } \\
\text { diffusion. }\end{array}$ \\
\hline $\begin{array}{l}\text { SensaBubble [94] } \\
\text { A device that generates scented } \\
\text { bubbles filled with fog. A } \\
\text { visual display is projected onto } \\
\text { the bubble and a scent released } \\
\text { when the bubble is burst. }\end{array}$ & $\begin{array}{l}\text { A controlled } \\
\text { mixture of three } \\
\text { base scents using } \\
\text { heated vaporisation } \\
\text { scents. The solution } \\
\text { for the bubbles } \\
\text { consists of water, } \\
\text { glycerine and } \\
\text { dishwashing liquid. }\end{array}$ & Research device & $\begin{array}{l}\text { Computer controlled } \\
\text { delivery of the scented } \\
\text { bubbles. }\end{array}$ & $\begin{array}{l}\text { Bubble } \\
\text { delivery } \\
\text { method. }\end{array}$ \\
\hline $\begin{array}{l}\text { Cyrano } \\
\text { Personal digital scent speaker. } \\
\text { Plays a medley of scents or } \\
\text { olfactory notes, aimed at } \\
\text { calming the mind and body. }\end{array}$ & $\begin{array}{l}\text { One scent cartridge } \\
\text { with a palette of up } \\
\text { to } 12 \text { scents. }\end{array}$ & \begin{tabular}{|l|} 
Commercially \\
available: \\
http://www.onotes.com
\end{tabular} & \begin{tabular}{|l} 
Scent emission \\
intensity is controlled \\
from the oNotes app. \\
However, currently only \\
supported with iOS \\
devices.
\end{tabular} & $\begin{array}{l}\text { Airflow } \\
\text { delivery } \\
\text { mechanism }\end{array}$ \\
\hline $\begin{array}{l}\text { Scentee } \\
\text { Personal scent emitting device } \\
\text { that can be attached to mobile } \\
\text { devices via the earphone jack. }\end{array}$ & One & $\begin{array}{l}\text { Commercially } \\
\text { available: } \\
\text { http://scentee.com/ }\end{array}$ & $\begin{array}{|lrr|}\text { SDK to } & \text { provide } \\
\text { programmatic } & \text { control } \\
\text { through } & \text { Android } & \text { and iOS } \\
\text { apps. } & \text { Supported } & \text { on iOS } \\
\text { and some } & \text { Android } \\
\text { devices. } & \\
\end{array}$ & $\begin{array}{l}\text { Combines } \\
\text { atomisation } \\
\text { with airflow } \\
\text { delivery. }\end{array}$ \\
\hline $\begin{array}{l}\text { Exhalia Diffuser SBi4 } \\
\text { Personal scent emitting device } \\
\text { connected via USB. Exhalia } \\
\text { also provides a range of other } \\
\text { scent emission devices to } \\
\text { support scented atmospheres, } \\
\text { point of sale and scented } \\
\text { objects. }\end{array}$ & $\begin{array}{l}\text { The SBi4 has the } \\
\text { capacity for four } \\
\text { scented porous } \\
\text { material cartridges. }\end{array}$ & $\begin{array}{l}\text { Commercially } \\
\text { available: } \\
\text { http://www.exhalia.com }\end{array}$ & 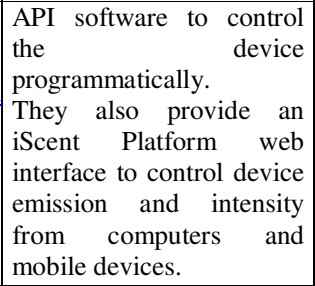 & $\begin{array}{l}\text { The SBi4 } \\
\text { uses the air } \\
\text { flow method. }\end{array}$ \\
\hline $\begin{array}{l}\text { Dale Air Vortex Activ } \\
\text { Personal scent emitting device } \\
\text { connected via USB. }\end{array}$ & $\begin{array}{l}\text { Four scented porous } \\
\text { material cartridges } \\
\text { can be loaded at a } \\
\text { time. }\end{array}$ & $\begin{array}{l}\text { Commercially } \\
\text { available: } \\
\text { http://www.daleair.com }\end{array}$ & $\begin{array}{l}\text { Scent control is via SDK } \\
\text { software program } \\
\text { provided with device. You } \\
\text { have the ability to control } \\
\text { the duration of the emitted } \\
\text { scent, but not its intensity. }\end{array}$ & Air flow \\
\hline
\end{tabular}


Yanagida [18] mentions five main methods of delivering vaporised scents to the target, with the method used largely depending on the scenario. Factors to consider include how many target users, are the users static or mobile, how many smells need to be delivered and the duration of the emitted scent(s). The natural diffusion/convection delivery method diffuses scents naturally diffused from a highconcentration area to a low concentration area and is more suited for ambient, background scented displays. The air flow method uses artificially generated wind to deliver a controlled emission of the scent to the user. It is one of the popular delivery methods being used, with a number of devices, such as Exhalia's Scent diffuser SBi4 and Dale Air's Vortex Activ using fans to generate the wind flow. The vortex ring approach [89] makes use of an air cannon to create a vortex of scented air. Tubes are also used as another delivery method to provide scented air just within the vicinity of the user's nose [18]. This approach has the advantage of the scented air not being exposed to odour diffusion and odour mixture suppression, but to avoid odour mixture suppression it means one scent per tube should also be used. The direct injection method, which involves directly injecting small droplets of liquid odorant into the user's nostrils is not suitable for controlled scent emission.

Despite the variety of vaporisation and scent delivery methods, there is still a limited availability of suitable and affordable computer controlled scent emission devices. This has been hampering research efforts in the area of olfactory displays. Recent research efforts are seeking to address this issue by creating open source scent-controlled devices [88], [90], making the details of both the device and odorants available to other researchers. Table I shows some of the more recently available devices. Some other notable devices that have been developed in the past include Scent Dome by TriSenx, iSmell by DigiScent, Osmooze, AromaJet, ScentWave by ScentAir and Scent Collar [91], [92]. This is not an exhaustive list, with greater details and comparisons available in [16][17][105] for the interested reader.

\subsection{Effects of intensity and duration of olfaction}

It is assumed that intensity and duration of olfactory component presentation will have a significant effect on user QoE. Due to the limitations of olfactory displays, efforts to understand these influencing factors have proven challenging for user QoE researchers. Initial studies exist that have relied on fan speed as a function of intensity, which have demonstrated the likelihood of proving the aforementioned assumptions but a more concrete understanding of the relationship between intensity, duration and resultant user QoE is a key step to the successful realization of olfaction-based mulsemedia experiences.

\subsection{How can we use olfaction in health, education, tourism, quality of life, storytelling etc.}

Notwithstanding each of the aforementioned challenges, a fundamental research challenge is to develop and understand the context of where and how olfaction based mulsemedia experiences can be exploited across a number of application domains. This requires a truly multi-disciplinary approach including but not limited to: chemists, neuroscientists, psychophysicists, educationalists, psychologists, artists, perfumers, historians, etc. to converge and collaborate with respect to the various application domains where olfaction-based mulsemedia applications are possible. Within each of these application areas, context-based QoE evaluation is required. 
Table II Summary of Reported Methodological Components in Olfaction-based Mulsemedia Studies

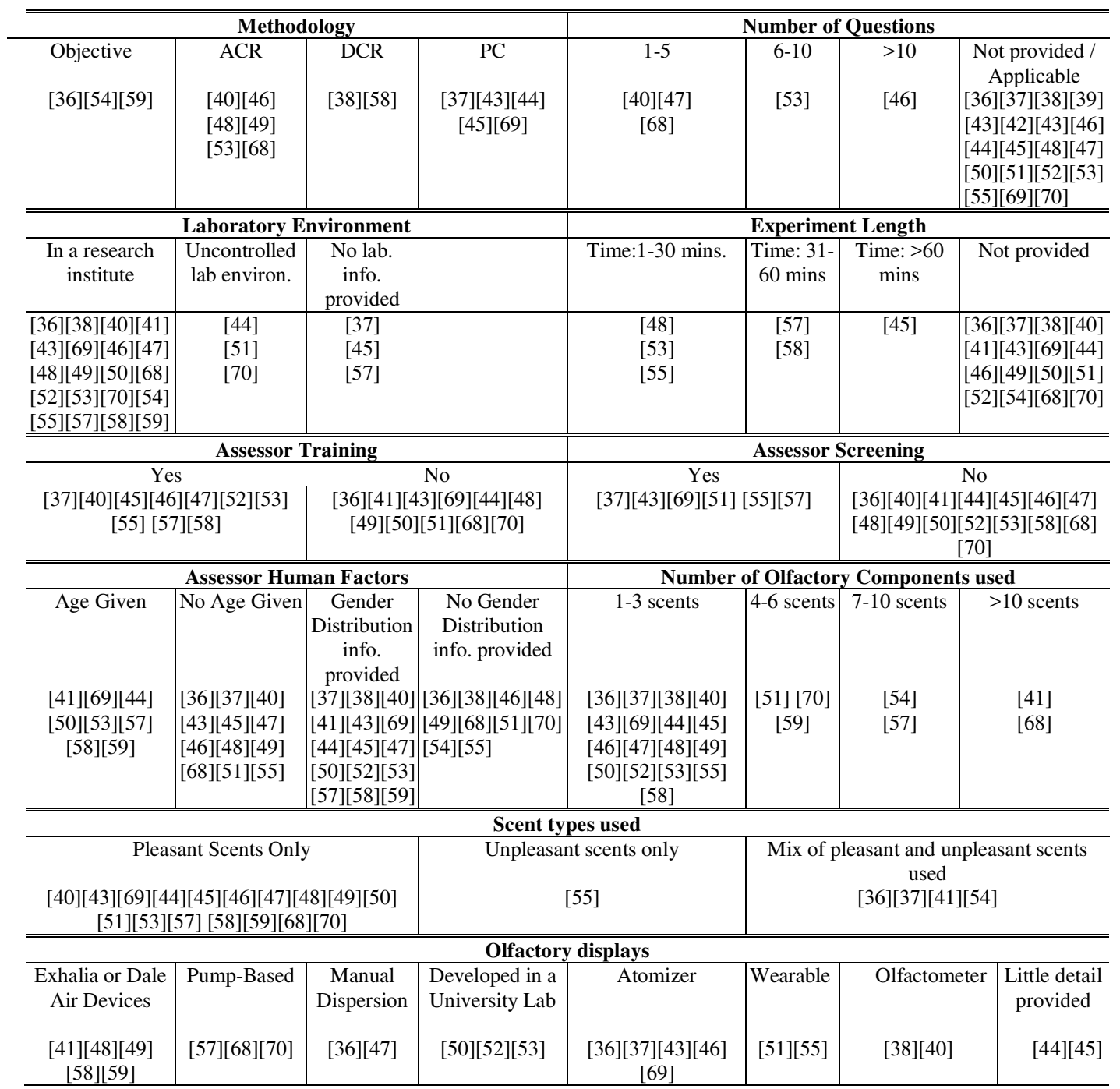

\subsection{Remote delivery of mulsemedia components}

Multimedia has traditionally been understood to be made up of loss tolerant, delay intolerant media; moreover, the bulk of the media being transported over communication networks has been continuous media (i.e. video). Unsurprisingly, this has spawned a wealth of research into communications protocols appropriate for transporting media with these type of characteristics [73][74][75]. However, mulsemedia components have different characteristics; indeed mulsemedia potentially comprises both traditional and non-traditional media, and novel 
communication protocols must be devised to transport not only non-traditional media (the information representations discussed in section 2.3) but also combinations of these with traditional media.

A greater understanding of the tolerance of non-traditional media to traditional multimedia issues of loss, delay, jitter and synchronization helps in this respect, as does clarity on what (meta)data needs to be transported in order for mulsemedia devices to play content appropriately. Nonetheless what constitutes an appropriate transport protocol for mulsemedia remains an open question.

It is therefore of paramount importance for the success of mulsemedia content and applications in general and olfaction-enhanced mulsemedia in particular that research and development effort be put in addressing these challenging aspects and make innovative proposals for further advancement of the state of the art.

\section{EXISTING STATE OF THE ART REVIEW}

In this section, the authors provide a summary of the different approaches to subjective evaluation of olfaction-based mulsemedia. It considers: methodology, laboratory environment, number of scents incorporated in tests, types of scent types used, length of subjective evaluation, number of assessors and assessor balance with respect to age and gender. In some of the works reviewed several of the above aspects were not reported. The findings are summarized in Table II. In addition, we present and critique these diverse approaches including own works, across each of these factors for olfaction-based mulsemedia evaluations. An overview

Anderson et al., [36] captured user perception of the olfaction-based mulsemedia experience through quantitative measurement using Electromyography (EMG). The multisensorial media system included a virtual reality head mounted display, with a platform for vestibular feedback and fans for the somatosensory stimuli. The olfactory component was manually dispersed using atomizers. Three scent types were used: pine, wet and horse, balancing between what could be termed pleasant or unpleasant scent types. No information was provided in the paper about the number of assessors, assessor training or screening, or lab environment.

Arroyo et al., [37][29] performed a study that analysed the effect different modalities had as interruption mechanisms. Two scents (soy sauce and Elmers glue) were presented to an assessor group of 12 which had a gender distribution of 8 females and 4 males. The effects of olfaction, heat, sound, vibration and light were considered. The olfactory display was an atomizer. As with the previously outlined work, no information was provided on the laboratory environment. In terms of training, assessors were informed that they would be tested about their reading performance. As part of preparation assessors were provided with scenarios which mimicked the actual tests i.e. a reading task accompanied by multimodal interruptions. In terms of screening, subjects with similar performance levels were selected after a reading and comprehension pre-test. However, no screening was reported with respect to assessor's olfactory capability.

In [38], the authors analysed the impact of olfactory adaptation on an assessors' ability to detect odors. The findings from works such as this highlight the requirement for a standardized approach to olfaction-based multisensorial media evaluation. The DCR or Degradation Category Rating [39] was employed to evaluate 
adaptation. The authors provided detailed information of the test lab, a mobile environment. The air in the laboratory, waiting and apparatus rooms and test booth was conditioned and purified. The temperature and humidity were maintained throughout the procedures. An olfactometer capable of controlling the concentration of the two odors was used for the scent presentation. No assessor training or screening was reported. A small user sample size of just 4 contained 2 males and 2 females. Given the context of this work, it is interesting to note that the article concluded by highlighting the importance of considering olfactory adaptation during for subjective evaluations.

An analysis was reported on the usefulness of various output modalities (text, olfaction, audio etc.) as notification mechanisms in [40]. The experiment contained 5 questions with answers graded on a Likert scale as well as an open interview. The inclusion of an open interview is unusual in multimedia evaluation, but was justified considering the variable perception of olfaction. There was a sample size of 12 , with a distribution of 10 females to 2 males. Two scents were used: cloves and eucalyptus. The olfactory displays were two Spa Scenter diffusers. In terms of training, before the start of the experiment, the participants were introduced to the experimental interface and were provided information on how to use it. The participants were told that they will have to engage and work on arithmetic questions whilst being presented with different types of notifications. They completed a training phase, where they answered arithmetic questions with no notifications. The training phase data was used as "a basis of comparison to experimental blocks that contained notifications" (i.e. control vs experimental analysis).

The usefulness of olfaction as part of searching digital photo collections was presented in [41]. The motivation for this study was based on the premise that an association between scent, memory, and emotion exists. The research question analyzed was if an olfactory component could be a useful cue for recall. The experiment compared text and smell based tagging. There were 12 assessors (4 females and 8 males) with an age range from 20-45 with a varied cultural background. A total of 16 scents were used in the tests with a mixture of pleasant and unpleasant scents: Brewery, Sweaty Feet, Riverbank, Unisex Perfume, Alpine, Smoke, Farmyard, Floral, Dusty, Bread, Sea Breeze, Sea Shore, Grass, Ozone, Machine Oil, and Dark Chocolate. The olfactory display employed was the smell cube from Dale Air [42]. The laboratory environment had two doors - which the authors stated supported good ventilation to the room (to avoid the problems of smell mixing). Whilst the authors provided no information on screening, their conclusion highlighted the requirement for screening programs for subjective evaluations involving olfaction.

Researchers also analysed the hypothesis that the presence of olfactory component could mask users' sensitivity to reductions in video quality and reported their findings in [43]. A pair comparisons [39] methodology was employed with high quality video and olfaction the control, and lower quality video with olfaction the test sample. The sample size was 66 with 19 females and 47 males with an age range from 18 to 57 years. The test environment included an empty room with a PC on a desk. Assessors were seated approximately $60 \mathrm{~cm}$ from the olfaction and video presentation system. The olfactory display was an off-the-shelf perfume atomizer which presented the scent of cut-grass. Although no detail of the screening process employed was reported in the paper, it was stated that all subjects "reported normal 
or correct to normal vision" [43]. In addition, none of the assessors reported any smell-related health problems (e.g. assessors having an allergy, a cold, or being pregnant). The paper also mentioned that the assessors had a basic level of knowledge of computer graphics. In [44], a large sample of 592 assessors (control group control group (447) / test group (145)) was employed, with an excellent balance across the age and gender variables. They were presented with one pleasant scent (citrus odor) as part of an experiment to determine the effects an ambient odor has on a shopper's spending. The key difference between the control group environment and test group environment was the presence of the odor. The environment for the test was a shopping mall. 10 scent diffusers were employed due to the large area in which the test took place. Their aim was to maintain consistent scent intensity. It was not stated how this was measured. No assessor screening or training was reported.

A research team focused on olfaction-enhanced learning assessment by employing Smart Ambience for Affective Learning (SAMAL) [45]. SAMAL is an ambient environment that integrates cognitive and affective approaches for learning. 80 assessors took part in the study. They answered questionnaires pre- and post-test. The balance in terms of gender was 22 males and 58 females. 2 pleasant scents, violet and apple green were used. In terms of training, the basic concepts of the tasks were explained. The time taken for this experiment was 90 minutes. In [46], in order to understand the sense of presence for users in a virtual environment on a per modality basis; a mulsemedia environment containing the effects of olfactory, tactile, visual and audio was evaluated. The assessor's ability to recall information on aspects of the environment was captured via 14 questions. The sample size was 322 , but no information on gender or age balance was given. One scent only (smell of coffee) was used. The laboratory environment was set up in a research institute. Olfaction was delivered to the assessor via an oxygen mask. No information on assessor screening was provided, however in terms of training, the participants experienced a virtual environment get familiarized with virtual environments. A System Usability Scale (SUS) questionnaire with 4 questions and answers to be graded on a five-point Likert scale was used to evaluate the effectiveness of olfaction as an input for second language learning [47]. A sample size of 12 (10 males and 2 females) were presented with a pleasant scent (Fresh mint leaves and stems (Mentha Spicata)). The laboratory environment consisted of a laptop with a 15.4" display in a computer room. The olfactory component was presented by participants rubbing mint leaves together, which released the odor as they were interacting with the virtual environment. In terms of training, the purpose and procedure of the test was explained. No screening was performed (or reported at least) and the experiment lasted 15 minutes.

The research reported in [48] employed 15 assessors between the ages of 21-29 in a university lab environment and graded their responses using the MOS. One scent (smell of rose) was presented using the SyP@D2, PHANToM Omni from Exhalia. The aim was to evaluate the influence of the source of the scent moving (direction and speed) on user perception of timing of scent release. The experiment lasted 15 minutes. Another work with a similar aim by the same authors employed 20 assessors between ages of 21-30 in a university lab environment [49]. One scent (smell of grapefruit) was presented using the SyP@D2, PHANToM Omni from Exhalia. The multisensorial media system also delivered haptic, audio and video components. 
In [50] users were required to identify the location of the scent source as part of understanding the effect of airflow on the perception of odors. 14 university students between ages of 21-26 (all male) took part in a university lab environment-located experiment. One scent (peach tea) was presented using the SyP@D2, PHANToM Omni from Exhalia [32]. The multisensorial media system also included haptic, audio and video. In [51] a novel system which stimulated the visual, olfactory and gustatory senses was developed. 43 assessors (no user profile information was provided) were presented with 5 scents (chocolate, almond, strawberry, maple, and lemon) as part of the mulsemedia system that aimed to trick assessors by conflicting their visual with their olfactory and gustatory senses. In terms of screening, the paper stated that participants had no expertise in anatomy. Assessors were not told about the aim of the experiment. The mulsemedia display system was "an air pumptype head-mounted olfactory display". In [52] 21 assessors with 17 males and 4 females were evaluated in terms of their reaction times to the presentation of 1 olfactory component.

The lab environment was a large university laboratory, approximately 70 square meters in size. This included multiple experimental spaces of $3 \mathrm{~m} \times 3 \mathrm{~m}$. Each were separated by partitions. The lab environment for also included two deodorizing apparatuses. Interestingly, the researchers controlled the position of the assessors' olfactory field - there were required to "place their chin on a chin rest". As such, the researchers were accurately able to measure and state that the distance from the device to the users olfactory field (i.e. their nose) was $22.5 \mathrm{~cm}$. In terms of training, assessors were instructed to control their breathing in line with an auditory cue. In [53] a comparison Likert scale was employed for answers to 6 questions which evaluated a scent ejection technique. 22 subjects, all in their 20 s, included 18 males and 4 females. 3 scents were used: lemon, cinnamon and heliotrope. In terms of training, assessors became familiarized with the three scents so that they could distinguish them to the point where if two scents were presented simultaneously they could detect and identify them. In order to prevent olfactory adaptation, there were approximately 30 -sec intervals between the trials, and subjects were instructed to take a break for around 5 minutes after every 8 trials. In [54] 7 scents (lemon, cookies (incense stick), cigarette, apple, coffee and curry) were used as part of the evaluation of an olfactory display built into the screen. Skin conductance was used to measure assessor's level of excitement. No information on number of assessors, assessor balance, training or screening was provided.

In [55], a sample size of 16 was presented with 1 scent as part of a virtual experiment (VE). In this work, "it was hypothesized that scent presentation during the VE would significantly improve recall" [55]. The objective metrics of heart rate and electrodermal activity (EDA) were measured during the experiment and interestingly the authors stated that EDA was a strong indicator of the ability to recall information. The scent used was a mixture of oil-based fragrances that matched the other content in the environment: a swampy culvert. The paper provided excellent detail on the hardware components used for visual and auditory stimuli. Subjects used a "Logitech Wingman cordless gamepad controller" for navigation in the environment. The lab was in a university. During the experiment, assessors were seated. The olfactory display used was a wearable device, named the scent collar [56]. In terms of training, subjects were shown an unscented system to help with familiarization purposes. Assessors were given 4 minutes to interact with 
the environment, and were not informed they will be asked to recall aspects later on. Testing time was 4 minutes plus the time to answer the questionnaire.

The research reported in [57] used a 16 point hedonic scale with a range from "very unpleasant" to "very pleasant" to rate 7 scents: Benzoin, Cashmera, Forest-Plus, Muguet, Peppermint, Sandiewood, and Spiced-Apple. Each scent was delivered by pumping air into a charcoal filter and then into a reservoir which contained the scent. The paper reported that assessors were screened for allergies and tested for anosmia and correct to normal vision. Assessors were also given an oxygen mask for familiarization purposes. Further, assessors were requested not to wear any deodorants. The authors reported that the experiment took 40 minutes to complete.

The research reported in [58] has employed the Degradation Category Rating (DCR) methodology with a sample size of 27 (14 males and 13 females). The age distribution reported 20 out of the 27 participants between 18-30 years with the rest between 31-60. 3 scents were used: chocolate, raspberry and riverside. The tests were carried out in a university lab, with the Vortex Active from Dale Air [42] as olfactory display. In terms of training, "at the beginning of each trial, participants were given an information sheet, a consent form and a short demographic survey. Participants were also asked to self-assess their sensory abilities on a 21-point Likert scale" [56]. Each notification and its association with the right button was explained to assessors. "Notifications were then delivered randomly until the subject had correctly acknowledged 6 sequential notifications" [56]. Participants were provided with corrective feedback each time an error was made. This ensured that each subject "had fully understood the links between notifications and buttons at the start of the game" [56]. When the games were finished, the users were required to complete a "paper-based NASA-TLX form" [56]. The experiment lasted 50 minutes.

The testing described in [59] involved 5 participants, all right handed males between the ages of 26 and 32 years. Assessors were requested to rate odors in terms of pleasantness (unpleasant to extremely pleasant - scale 0-10) and intensity (no odor present to intolerable for intensity - scale 0-10). Four scents were presented: "valerian, lotus flower, rosewater and fermented goat cheese" [59].

In terms of assessor training, assessors were not told what odors that would be presented but were given high level information on the purpose for the experiment. Assessors were also requested not to wear odorant products on the day of the test. Participants were screened via a questionnaire for any respiratory, mental or chronic disease. The paper also reports that olfactory adaption and assessor fatigue were considered in defining a minimum of 4 seconds between trials employing the same odor.

\subsection{An overview from the experiences of the performing olfaction-based mulsemedia QoE evaluations}

With respect to our own experiences of performing olfaction-based mulsemedia QoE evaluations, here we highlight the approaches taken in [1][8][16][17][22][25][26][27][28][28][30][31][33][34][35]. Details about the laboratory design, olfaction-based mulsemedia presentation equipment, assessor numbers and profile, screening of assessors, subjective testing approach, questionnaires and rating scales are presented.

Three similar laboratory designs were used: in Athlone Institute of Technology in Ireland, Brunel University in UK, and Dublin City University, Ireland. The 
laboratories used for [1][17][22][25][26][27][28] were designed in accordance with [62] such that it enables:

- performing assessment in controlled and known conditions with minimum distraction

- reducing physical condition and psychological factor effects on human judgment.

The lab in Athlone Institute of Technology, Ireland is presented in Fig. 1. It includes room A as a preparation and sample storage room, room B as an experimentation room, and room $\mathrm{C}$ as a test subject waiting room. The test room walls are painted Matt off-white. The testing booth is situated in the test room corner in order to minimize distraction (Fig. 1 B1) and the questionnaires were as far away as possible from the testing booth (Fig. 1 B2). This allowed time for scents to diffuse, minimized adaptation, as well as gave assessors a break between each judgment. It also prevented the subjects from being influenced by lingering scents. A sign was posted on the door to ask any subject to wait outside until invited in. The assessors did not have any access to the preparation and storage room. Whilst there was no specific ventilation system, the test lab was large, had 3 doors and many windows to allow scents be removed after the tests.

For the studies reported in [28][30][31][33][34][35], the experiments were conducted in Brunel University in the UK. As per Fig. 2 the laboratory had one door and one large window. The door and window were left open before and after the experiment to ensure any ambient odors present in the room were removed. The participants were seated on one side of the laboratory and were using individual computers for multimedia video clip display.

The experimental studies reported in [8][65] came from the Dublin City University-based Performance Engineering Lab, Ireland (PEL@DCU) as illustrated in Fig. 3. The room had two windows which were closed for the duration of the tests in order not to have outside atmospheric disturbance to influence the tests. The windows were open in between the tests in order to allow the outside fresh air to help any remaining lingering scents to diffuse and enable new tests to take place in neutral conditions. The Preparation Desk was needed to prepare any test materials in advance of any new round of tests. The test subjects were asked to wait outside the test room until they were called in. Test details were explained to them while sitting at the Information Desk and once all potential test aspects were clarified, testing started at the Testing Desk, located at the right furthest away from door corner. All testing conditions suggested in ITU-T R. P.910 [21], ITU-T R. P.911 [66] and ITU-T R. P.913 [67] were complied with. 


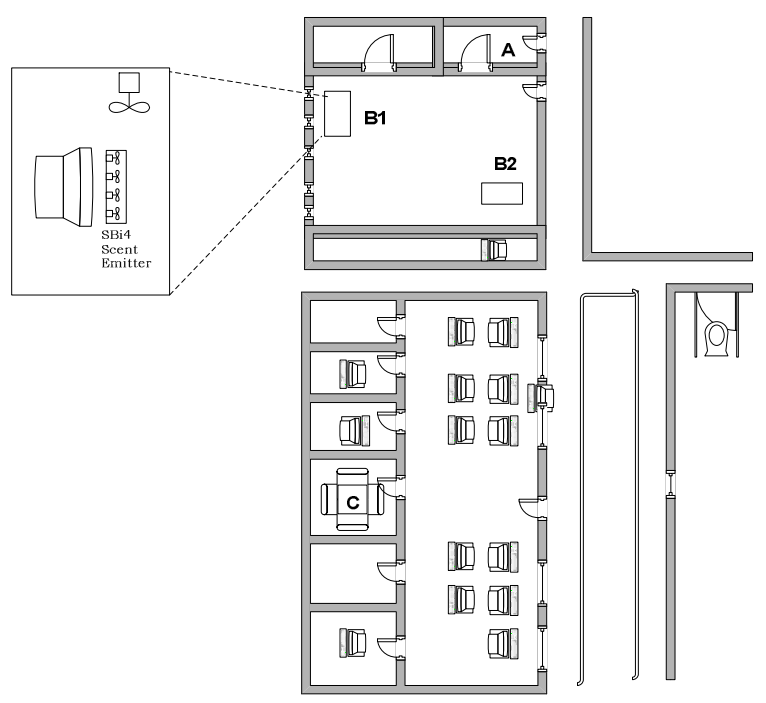

Fig. 1: Athlone Institute of Technology Olfaction based Mulsemedia Lab: Plan View of Experimentation Room (B), Preparation room (A) Meeting room (C). Also shown is the desk where assessors participate in the tests. (B1) [1]

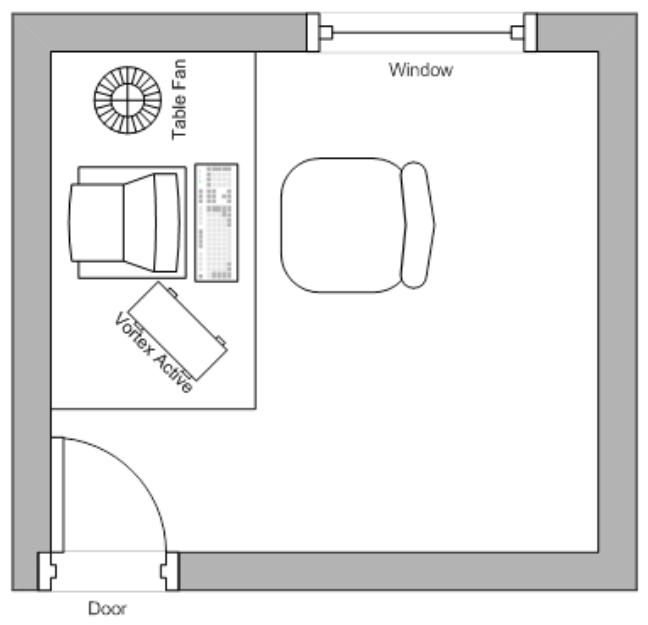

Fig. 2 Brunel University Olfaction based Mulsemedia Lab: Plan View of Experimentation Room [33] 


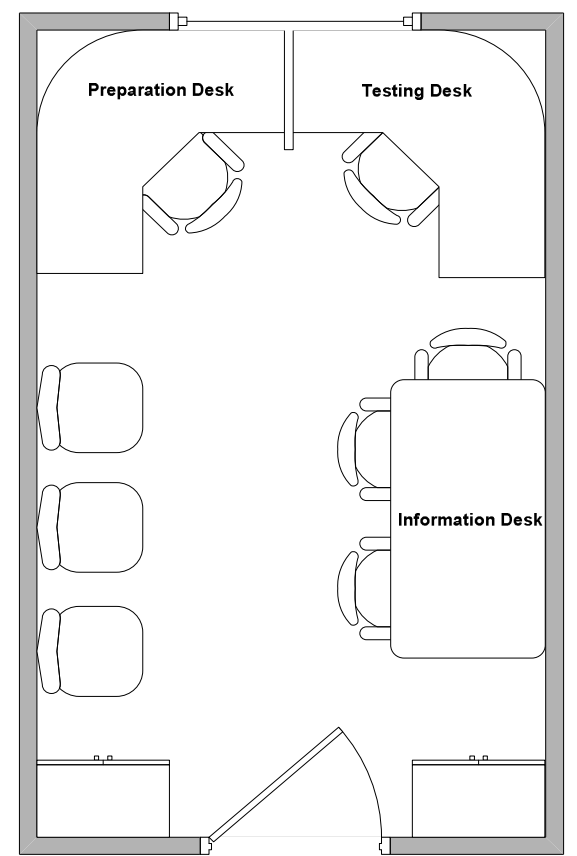

Fig. 3: DCU-located olfaction-enhanced Mulsemedia perceptual test-bed [8]

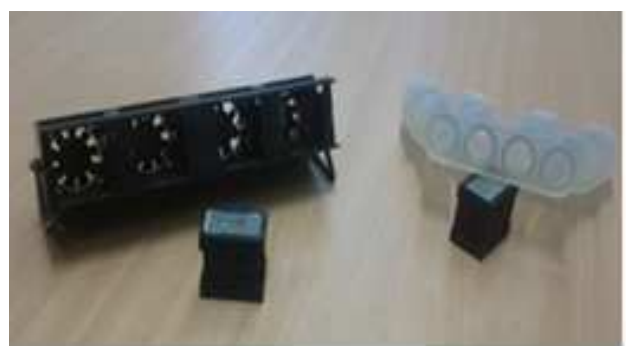

Fig. 4a: SBi4 V2 from Exhalia used in AIT experiments [22]

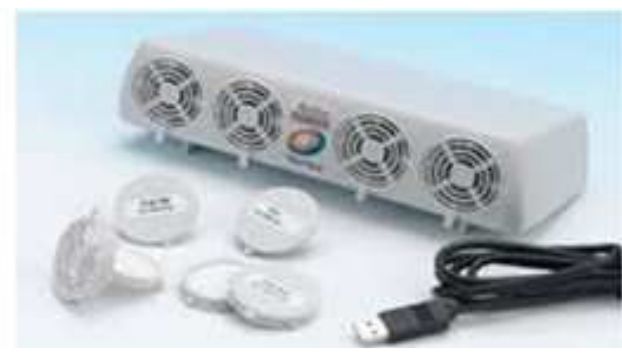

Fig. 4b: Vortex Active from Dale Air in Brunel University and Dublin City University studies [28]

The olfactory displays (OD) used across the 3 test sites were: the SBi4 - radio v2 olfactory display from Exhalia [32], presented in Fig. 4a and the Vortex Active from Dale Air [42], shown in Fig. 4b. Both ODs had very similar operating principles. They used 4 in-built fans to present scents by blowing air through scent cartridges. Both ODs allows control of the intensity of scent emission by changing the fan speed. During the tests the maximum fan speed was used. In the Vortex Active, the scent cartridges were based on cotton pads soaked in scented oil whereas the cartridges for the SBIx were made from scent polymer balls. Considering a distance of 0.5 meters between $\mathrm{SBi} 4$ and assessor, it was experimentally determined that it took between $2.7 \mathrm{~s}-3.7 \mathrm{~s}$ for users to detect the various scents. It took on average 2 seconds for scents to be detected from the Vortex active device. Both olfactory displays were 
controlled by SDKs with the devices connected a laptop via a USB port. Special control programs were developed to control the presentation of olfaction-based mulsemedia, as outlined in detail in [25][26] [28].

The assessors were screened as per ISO 5496 standard [64]. This standard defines how the initiation and training of assessors for detection and recognition of odors should be performed. Among others, this helps teach assessors to:

- Evaluate if they could perceive the presence of an odor

- Identify odors

- Use appropriate vocabulary.

However this standard was also employed in order identify assessors who may have anosmia, i.e. lack of sensitivity to certain scents. The process of pre-screening involved assessors being presented with scents and asked if they could:

1) "perceive an odor" [64]

2) "recognize an odor" [64]

3) "name the odor presented" [64].

Also in terms of training if an assessor was able to detect, but not identify a particular odour, they were told the name of the odor.

To evaluate human perception of multimedia experiences, multiple subjective rating methods and subjective metrics have been proposed and standardized in the past. We have employed two of these methodologies, namely Absolute Category Rating (ACR) and Degradation Category Rating (DCR) from ITU-T P.910 [21]. Employing ACR in [8][28][31][34][35][65], participants were presented with one olfaction-based mulsemedia sample and were asked to provide their level of agreement with statements in the questionnaires. In [1][22][25][26][27][28] DCR was employed. With this approach, assessors were presented with two olfaction-based mulsemedia samples. The first always had optimum quality (known as the reference) and a second sequence had a certain level of impairment, known as the sample under test. Assessors rated the quality of their experience against the questionnaire's Likert scales.

Six experimental questionnaires were designed and employed during the research studies previously reported by the authors. The questions were designed to gather data across the various parameters that could have an impact on user QoE of olfaction-based mulsemedia i.e. skew, scent type, video content, and information recall. As part of the preliminary testing to ensure the content of the questionnaires was clear, a reliability assessment was undertaken. Discussions with the subjects took place, and based on the feedback, amendments were made to the questions. A psychologist has also reviewed the final question list.

During the olfaction-based mulsemedia evaluations, at the end of each test sequence, assessors completed a questionnaire on their experience. The questionnaires comprised of statements used to request participants' opinions in respect of the olfaction-based mulsemedia clips presented. The assessors had to grade their answers in each test question using the 1 to 5 Likert scale. The specific content of the questions are available in [26][25][28]. The authors accept that the content of some of the questions may have had a positive bias.

The entire test time for a single subject participating in the tests conducted at the Athlone Institute of Technology was 1 hour [1][22][25]. This involved "250 seconds per test sequence (i.e. reference sample, break, sample under test and voting)" [26] and a 10 minute break once 30 minutes elapsed in the assessment. For the study 
reported in [26] the testing time for a single subject was approximately 65 minutes. This involved 350 seconds for each test part. There was a break at the mid-point of each of the tests with olfactory adaptation or assessor fatigue in mind. The test subjects were allowed to drink water when completing the questionnaires, but not during the presentation.

The time for a single subject case for the works performed at Brunel University and reported in [28][30] was approximately 30 minutes. This comprised of approximately 300 seconds per test sequence (sample under test and completing the questionnaire for the sample under test). Allowing assessors to respond to the questionnaire for the sample under test after each test sequence ensured there were breaks between the delivery of the olfactory media and also served to address concerns over olfactory adaptation. Assessors resumed the next test sequence when they were ready, thus addressing concerns over assessor fatigue.

The Dublin City University testing time was no longer than 30 minutes per volunteer [8][65]. This comprised of watching 16 clips of 30 seconds each and time to answer relevant questions after the visualisation of each such olfaction-enhanced sequence. The subjects were asked to leave if they would suffer from fatigue of any other effect which would negatively influence their performance. Assessors were not permitted to consume any food or drink immediately before and during the testing.

For the work reported in [1][22][25][28][33], the six videos used were of 90s duration and are presented in Table IV. In each video, the middle 30 second segments contained the video content relevant to the olfactory component. The clips included cookery programs, documentaries and news shows. The scents of "burnt, foul, fruity, flowery, resinous and spicy reflect a fair distribution between what can be termed as pleasant and unpleasant smell categories" [1].

For [26], eight videos of 120 s duration were used and are presented in Table IV. These video clips were divided into four $30 \mathrm{~s}$ blocks whereby the two middle $30 \mathrm{~s}$ blocks contain content related specifically to the scent being presented. These clips have also included cookery programs, documentaries and movies. These were chosen because they contained a balance of video content reflecting a mix of pleasant, unpleasant and scents that could be possibly considered pleasant or unpleasant. The scents of fruit, forest, flowery, burnt, chocolate, orange, horse stable, seawater and grass also reflect a fair distribution between pleasant and unpleasant smell categories. Ten different such scents were used in the testing. Hence all works comply with [64] in that neither exceeded the recommended maximum of 10 scents to be used in subjective tests. The scents were stored in sealable plastic bags. In addition, to ensure consistency in terms of concentration, they stored at approximately $5^{\circ} \mathrm{C}$, as recommended in [64].

In the Dublin City University tests, each assessor watched 16 of a pool of 32 multimedia sequences. These sequences were selected from the movies "Jurassic Park" and "Back To The Future". The clips were 30 seconds in duration. An olfaction component was integrated into 16 of the clips according to the sequence content scenarios, as given in Table V and Table VI. The other 16 had no olfaction content associated with them. Sample content was taken from the movie "Back to the Future". For the two movies, four video clips were selected with high motion content (video varies rapidly e.g. sport or action movie) and four with low motion content (video varies slowly e.g. talk show). These clips were shown to the test subjects in a random order. Regarding scent types, burnt, rubbish, methane, rock pools, mulled 
wine and forest were employed, reflecting a nice mix of pleasant and unpleasant scent types. These were selected in order to best match the video content in terms of realism.

A total of 350 assessors took part in the findings reported in [1][17][25][26][27][28], "between the ages of 19 to 60 years from a wide variety of backgrounds: students, academic staff, health care professionals, post graduate researchers, farmers, members of defence and police forces, accountants teachers, IT industry professionals, persons from medical and construction industry and also persons unemployed". The group included users from multiple cultures and nationalities. The studies reported in [10], [28][30][31], [33][34][35] involved a total of 173 assessors, made up of 89 males and 84 females from different cultures and nationalities. Assessors ranged from $18-41$ years of age and were from a wide variety of backgrounds, and socioeconomic groups.

The Dublin City University-based tests reported in [8][65] involved 16 users (i.e 9 males and 7 females). Participants were from different backgrounds, e.g., education, finance, engineering etc., in the 20-36 age range. No information on the cultural background was collected.

It can be seen how the three olfaction-enhanced mulsemedia QoE assessment testing sessions have many similarities, but also differ in many aspects. Similarities include the layout of the testing environment, presentation equipment (olfaction dispenser), assessor profile, screening of assessors, subjective testing approach, questionnaires and rating scales. However these testing sessions have differed in terms of the number of participants, number and content of the mulsemedia sequences and olfaction stimuli to which the participants were exposed to. To complete the end-to-end workflow for olfaction-based multimedia, we highlight our experience of working with the delivery of olfaction-based mulsemedia components.

\subsection{An overview from a mulsemedia delivery perspective}

Directly addressing research challenge 2.7 above, this section describes a generic architecture and presents several key issues regarding the design of an olfactionenhanced mulsemedia delivery system. Three critical aspects are discussed:

1) Overall system architecture

2) Olfaction-enhanced mulsemedia data packet header

3) Combination with other types of sensorial data.

\subsubsection{System architecture}

Diverse architectural detailed designs could be employed for the olfactionenhanced multisensorial delivery system. However, a generic client-server architecture is illustrated in Fig. 5. It includes a Sending Buffer, a Packet Scheduler, an Encoder (such as an MPEG-7 encoder for instance) and a Packet Delivery component on the Server side and a counterpart Decoder (e.g. MPEG-7) and Content Presentation unit at the Client side. Optional Adaptation and Delivery Monitoring and Feedback components can be present at the Server and Client, respectively, if mulsemedia content adaptation is envisaged [8]. Olfaction-enhanced mulsemedia content delivery will be performed over any IP network.

Indeed, whilst this architecture targets olfactory-based applications, architectures targeting other mulsemedia data have been proposed in the literature such as 
PlaySEM [95] and SEMP [96] and it is reassuring that most comprise classic multimedia blocks, namely content, distribution, rendering and QoE. Also classic (but of course, transposed to a mulsemedia context), are the nature of the problems and challenges encountered and outstanding within each of these modules. In terms of content, whilst the debate around mulsemedia storage is still ongoing, there seems to be agreement in the literature that, as regards (meta-) description, the two standards that should be employed are MPEG-V and/or MPEG-7. Mulsemedia distribution remains a challenge and is beset by the traditional multimedia issues of delay [98], synchronization ([1], [22]-[24], [31], [99], [101], [102], [103]), jitter [24], [31] and masking [100]. Rendering of new media types, such as olfactory and gustatory, in a digital (and distributed) context is, with a few exceptions [17] [104], still relatively unexplored. The success of any application is, unsurprisingly, inextricably linked to QoE; the main issue here - and which the current paper addresses - is that QoE evaluation methods for mulsemedia applications tend to be ad hoc as a direct result of a lack of accepted methodological standards by the stakeholder communities.

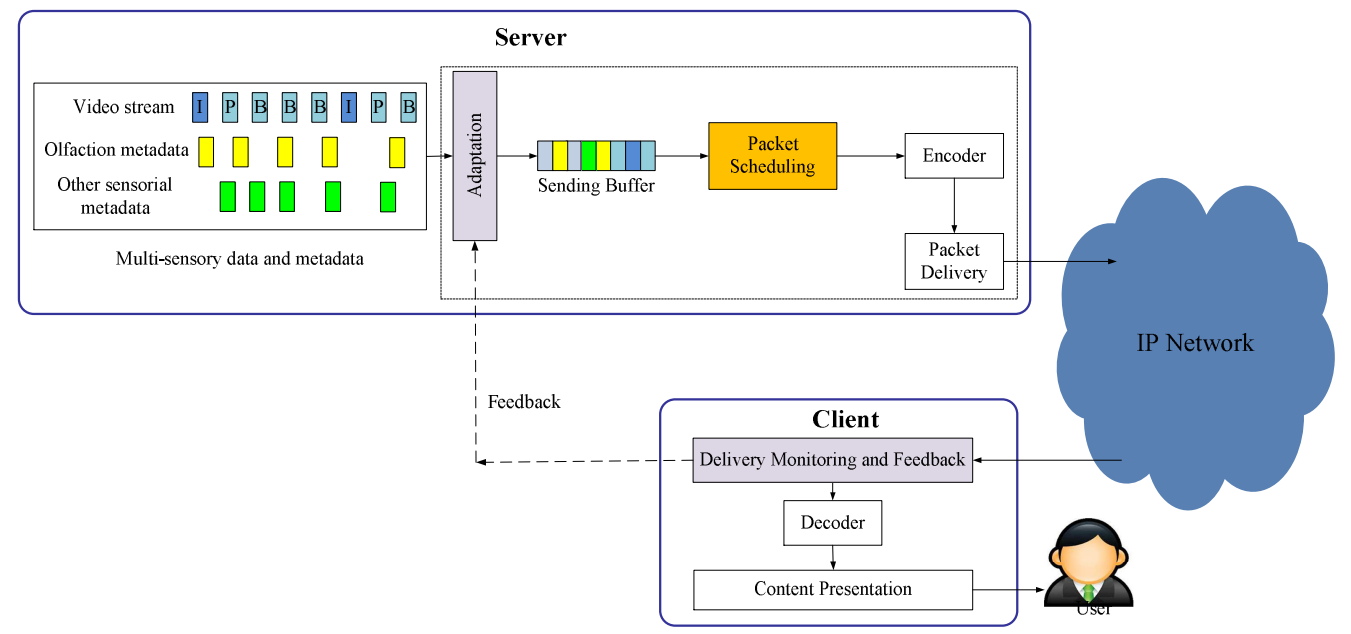

Fig. 5 Generic Architecture for an Olfaction-enhanced Mulsemedia Content Delivery System

\subsubsection{Olfaction-enhanced mulsemedia data packet header}

A special packet header for sensorial data is created to for the delivery of olfaction-enhanced mulsemedia packets in IP-based networks. For this delivery, typically the mulsemedia packets are created using the mulsemedia data packet header and then they are encapsulated into an existing codec (e.g. MPEG-7). The MPEG packets are then multiplexed and streamed over the chosen IP network. Such a mulsemedia data packet header for sensorial content in general and olfactionenhanced mulsemedia content in particular is described in Table III [8]. 
TABLE III

DESCRIPTION OF SENSORIAL DATA PACKET HEADER

\begin{tabular}{|c|c|l|}
\hline Name & Size & \multicolumn{1}{|c|}{ Description } \\
\hline $\begin{array}{c}\text { sequence } \\
\text { number }\end{array}$ & 2 byte & Identifies the sensorial data packet. \\
\hline type & 1 byte & $\begin{array}{l}\text { Sensorial effect type, e.g. olfaction, haptic, } \\
\text { etc. }\end{array}$ \\
\hline intensity & 1 byte & $\begin{array}{l}\text { Sensorial effect intensity, e.g. strong, } \\
\text { medium, weak. }\end{array}$ \\
\hline start time & 4 bytes & $\begin{array}{l}\text { Sensorial effect start time (used in } \\
\text { conjunction with } \text { duration for } \\
\text { synchronization with other effects) }\end{array}$ \\
\hline duration & 4 bytes & $\begin{array}{l}\text { Sensorial effect duration. (used in } \\
\text { conjunction with start time for } \\
\text { synchronization with other effects) }\end{array}$ \\
\hline option & 4 bytes & Extensible by users \\
\hline
\end{tabular}

\subsubsection{Combination with other types of sensorial data}

The olfaction component may co-exist with diverse other media elements including audiovisual, haptic, etc. These elements consist of either metadata only (i.e. olfaction) or both metadata and content (i.e. video). Metadata describes most sensorial effects to be presented remotely by various devices, after mulsemedia was delivered over the network. This metadata describing the different sensorial media components identifies not only their start time and duration, but also the intensity of the sensorial effect. There are some specific sensorial characteristics which require additional fields for the metadata including direction for air motion, flavor for the gustatory effect, and scent type for olfaction. The sensorial metadata is represented using well known standards like MPEG-V [19] and MPEG-7 [71].

However the most challenging issue when combining multisensorial components in the same mulsemedia stream and especially when delivering them, is to achieve certain temporal relationship between them: perceived zero intermedia skew. For instance, a perceived zero skew between the visual and olfaction components indicate an excellent temporal synchronization between them and is associated with the best user quality of experience levels.

This ideal inter-media synchronization is achieved by employing the metadata features: start time and duration, and a process of careful synchronization control during remote presentation. However, presenting sensorial media to users is not as simple as (dis)playing traditional multimedia (i.e. audio and video) and may cause less desirable user perception effects. For instance, the duration of smell may be perceived by users for a longer or shorter period of time than the originally intended one, due to effects such as lingering and propagation. Murray et al., [72] have added constant offsets and increased the time between different media presentations in order to address these issues. 
Table IV: Breakdown of video and scents used in [1][22][25][28][28][30]

\begin{tabular}{|c|c|c|c|c|c|c|c|c|c|}
\hline $\begin{array}{l}\text { Scent } \\
\text { Category }\end{array}$ & Burnt & \multicolumn{2}{|c|}{ Flowery } & Fruity & \multicolumn{2}{|l|}{ Foul } & \multicolumn{2}{|c|}{ Resinous } & Spicy \\
\hline \multirow[t]{2}{*}{ Clip No: } & Clip 1 & \multicolumn{2}{|c|}{ Clip 2} & Clip 3 & \multicolumn{2}{|l|}{ Clip 4} & \multicolumn{2}{|c|}{ Clip 5} & Clip 6 \\
\hline & $\begin{array}{l}\text { Documentary } \\
\text { about bush } \\
\text { fires in } \\
\text { Oklahoma }\end{array}$ & \multicolumn{2}{|c|}{$\begin{array}{l}\text { News } \\
\text { broadcast } \\
\text { about a } \\
\text { perfume } \\
\text { launch } \\
\end{array}$} & $\begin{array}{l}\text { Documentary } \\
\text { about the } \\
\text { process of } \\
\text { fruits rotting }\end{array}$ & \multicolumn{2}{|c|}{$\begin{array}{l}\text { Cookery } \\
\text { show on how } \\
\text { to make a } \\
\text { fruit cocktail }\end{array}$} & \multicolumn{2}{|c|}{$\begin{array}{l}\text { Documentary } \\
\text { about spring- } \\
\text { time allergies } \\
\text { and cedar } \\
\text { wood. }\end{array}$} & $\begin{array}{l}\text { Cookery } \\
\text { show on how } \\
\text { to make a } \\
\text { chicken } \\
\text { curry. }\end{array}$ \\
\hline \multicolumn{10}{|c|}{ Table IV: Breakdown of video and scents used in [26][28] } \\
\hline $\begin{array}{l}\text { Scent } \\
\text { Category }\end{array}$ & Fruit / Flower & $\begin{array}{l}\text { Forest / } \\
\text { Burnt }\end{array}$ & $\begin{array}{l}\text { Fruit } \\
\text { Rubbish }\end{array}$ & $\begin{array}{l}\text { Rotting / } \\
\text { Burnt }\end{array}$ & $\begin{array}{l}\text { Orange / } \\
\text { Chocolate }\end{array}$ & $\begin{array}{l}\text { Hor } \\
\text { Stak } \\
\text { Gra }\end{array}$ & I & $\begin{array}{l}\text { Forest / } \\
\text { Seawater }\end{array}$ & $\begin{array}{l}\text { Grass } \\
\text { Seawater }\end{array}$ \\
\hline Clip No: & Clip 1 & Clip 2 & Clip 3 & Clip 4 & Clip 5 & Clip & & Clip 7 & Clip 8 \\
\hline $\begin{array}{l}\text { Video } \\
\text { Description }\end{array}$ & $\begin{array}{l}\text { Documentary } \\
\text { about flower } \\
\text { gardens and } \\
\text { Orchards. }\end{array}$ & $\begin{array}{l}\text { Scene } \\
\text { from } \\
\text { the } \\
\text { Avatar } \\
\text { Movie. }\end{array}$ & $\begin{array}{l}\text { Docu- } \\
\text { mentary } \\
\text { rotting } \\
\text { fruit } \\
\text { cocktail. }\end{array}$ & $\begin{array}{l}\text { Scene } \\
\text { from } \\
\text { Lord of } \\
\text { the } \\
\text { Rings } \\
\text { Movie. }\end{array}$ & $\begin{array}{l}\text { Docu- } \\
\text { mentary } \\
\text { about } \\
\text { making } \\
\text { chocolate } \\
\text { orange } \\
\text { biscuits. }\end{array}$ & $\begin{array}{l}\text { Doc } \\
\text { men } \\
\text { abo } \\
\text { hor } \\
\text { stab }\end{array}$ & $\begin{array}{l}\text { ary } \\
\text { in } \\
\text { ing. }\end{array}$ & $\begin{array}{l}\text { Scene } \\
\text { from } \\
\text { Avatar } \\
\text { Movie. }\end{array}$ & $\begin{array}{l}\text { Documentary } \\
\text { about the } \\
\text { grass plant } \\
\text { and sea life. }\end{array}$ \\
\hline
\end{tabular}

TABle V: Olfaction EFFECT ATtACHED to the MUltimedia CONTENT FROM “JURASSiC PARK”

\begin{tabular}{cccc}
\hline \hline Motion & $\begin{array}{l}\text { Video } \\
\text { Clip Code }\end{array}$ & Movie Scenario & Olfaction Aroma \\
& & & \\
\hline \multirow{6}{*}{ High H1 } & Mild animal attack & None \\
& JP H2 & Severe animal attack & None \\
& JP H3 & Wind as car moving fast & None \\
& JP H4 & tear gas & Burnt \\
& JP H5 & Vehicle vibration and wind & None \\
& JP H6 & Animal attack and smoke & Burnt \\
& JP H7 & Wind and fire & Burnt \\
& JP H8 & Vehicle vibration, wind and forest & Forest \\
& JP L1 & Daily life & None \\
& JP L2 & Animal attack & None \\
JP L3 & Subway train comes & None \\
LP L4 & Decomposed animal odor & Rubbish \\
& JP L5 & Pull by parasail and wind & None \\
& JP L6 & Air plane and crash & Methane \\
JP L7 & Ocean wind and wine & Rock pools, Mulled wine \\
& JP L8 & Movement, gas and wind & Methane \\
\hline \hline
\end{tabular}

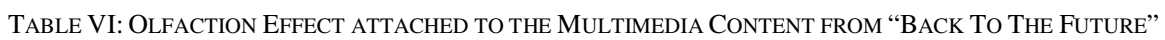

\begin{tabular}{cccc}
\hline \multirow{2}{*}{ Motion } & $\begin{array}{c}\text { Video } \\
\text { Clip Code }\end{array}$ & Movie Scenario & Olfaction Aroma \\
& & & \\
\hline \multirow{4}{*}{ High } & BF H1 & Bar scene & None \\
& BF H2 & Car crash & None \\
& BF H3 & Wind & None \\
& BF H4 & Smoke & Burnt \\
& BF H5 & Crash and wind & None \\
& BF H6 & Car crash and manure & Rubbish acrid \\
& BF H7 & Wind and smoke & Burnt \\
& BF H8 & Car movement, wind and smoke & Burnt \\
\hline
\end{tabular}

ACM Computer Surveys, Vol., No., Article, Publication date: 


\begin{tabular}{cccc}
\hline & BF L1 & Meeting friends & None \\
& BF L2 & Car crash & None \\
BF L3 & Wind & None \\
Low & Burning bread & Burnt \\
BF L4 & Falling down and wind & None \\
BF L5 & Sound waves and smoke & Burnt \\
BF L6 & Smoke and wind & Burnt \\
BF L7 & Car movement, fire and wind & Methane \\
\hline BF L8 &
\end{tabular}

This section has provided an overview of the state of the art, inclusive of the authors efforts from many of the factors relevant to olfaction-based mulsemedia. Next we present a set of recommendations in order to bridge the gap between the existing standards in terms of multimedia assessment and existing practical approaches for olfaction-enhanced mulsemedia assessment. In the next section, we critique these efforts highlighting diversity of approaches presented in the literature to capture user QoE of olfaction-based mulsemedia.

\section{STATE-OF-THE-ART CRITIQUE}

As illustrated in the previous section, they are differentiating aspects for olfactionenhanced multimedia quality assessment which include: methodologies to capture user QoE; number of questions in questionnaires; testing environment; training and screening process; participant age and gender; number of olfactory components used and scent type. Next, the studies reported are compared from each of these perspectives.

The methodologies employed to capture olfaction-based mulsemedia QoE can be classified broadly into two categories: post experience (explicit) and during experience evaluations (implicit). The post experience evaluations have evolved from ITU-T standards for audiovisual quality evaluations: ACR, DCR, and Pair Comparison (PC). The implicit evaluations are objective approaches to capture QoE via physiological metric capture (EEG, EDA etc.) and analysis performed. There has been a fragmented approach within each category driven by a lack of methodologies for olfaction-based mulsemedia evaluations. Timmerer et al., [20] reported that there were no statistically significant differences between the evaluation of sensory experiences captured via ACR and DCR. However, the researchers reflected that the most suitable assessment approach was based on the double stimulus continuous quality scale (DSCQS) method with minor modifications to fit the requirements of mulsemedia evaluations. They also concluded that ACR could be used without modifications, which is valid. However, during our own pilot testing, we noted the novelty effect of olfaction-based mulsemedia, whereby users were temporarily willing to accept degradations in quality. Moving forward, it appears that ACR is most suited however with the caveat that assessors have to undergo a significant training phase to address the novelty aspects, whilst also addressing non-uniform distribution of results as mentioned in [61]. Within lies the argument for a double stimulus approach (DCR/PC) - assessors can base their judgments on the test sample having also being presented with a reference sample. However, with respect to olfactionbased mulsemedia the double stimulus approach potentially raises some issues such as assessor fatigue and olfactory adaptation. 
Although not all experiments required a questionnaire, it is a little alarming that for almost $80 \%$ of experiments, no detail on questionnaires was provided, particularly for repeatability of research. With just 5\% asking more than 10 questions, it appears that large numbers of questions are not an issue. For those studies that exceed 10 questions, how to maintain assessor interest is challenging. Also considering the novelty and the number of unknown factors which influence olfaction-based mulsemedia, we also highlight the potential benefits for open-interview approaches to solicit information and quality evaluation from assessors.

The mulsemedia test environments previously reported in the literature. In almost $25 \%$ of the articles reviewed, the laboratory environment was either uncontrolled (e.g. in a public place) or no information was provided. On initial viewing, 75\% reporting their lab environments in a research institute seem commendable. However, significant differences existed between the labs reported. If we consider the strictly reported laboratory environment specified for video evaluations, as in [61], no olfaction-based mulsemedia equivalent exists. Specific to olfaction, the ISO 8589:2007 [62] standard reports a number of recommendations as discussed in section 5 of this paper. One key point for future work at a basic level is that in [61], the authors specified the walls should be a neutral "grey" color for video quality evaluations. However, in [62], for olfactory they specify the walls be "matt-off-white" and Timmerer mentions in [20] that black background is most suited to highlight mulsemedia effects. The question is if and how synesthesia [63] influences user perception across the different senses and its influence on $\mathrm{QoE}$ is a future research topic of merit. In addition, the laboratory environment should aim to ensure assessor comfort. It should exist a method to extract the olfactory components or at least have some method to address the lingering effects of scents. Finally, and ideally, there should be a method to capture and or control the user's olfactory field and maintain it throughout the olfaction-based mulsemedia evaluation process.

In terms of time taken to undertake mulsemedia evaluations, for over $70 \%$ of the works reported, no information was provided in the literature with respect to timing. However we believe, it is key to consider the effect of continued olfactory presentation especially considering users' ability to detect and perceive scents (i.e. olfactory adaptation). Steps should be taken to address this as it is discussed in section 5. A method to monitor and react to olfactory fatigue should be employed.

As part of the approach for olfaction-based mulsemedia evaluation, two key steps that should be considered are training and screening. The motivation for inclusion of both aspects is to ensure that:

(1) the novelty of olfaction does not skew results

(2) assessors familiarize themselves with olfaction-based mulsemedia

(3) subjects are healthy, able to conduct evaluations and offer repeatable results.

For example, olfactory anosmia is a phenomenon whereby a person has an inability to detect particular scents. In the literature, only $29 \%$ of the works reported screening of some sort. The training and screening methodologies should include the materials and content that the test scenarios employ.

In terms of consideration of human factors and the participants who have taken part in studies, the breakdown on gender analysis is positive with $51 \%$ male and $49 \%$ 
female reported as having taken part in the evaluations. However, this is only for the $40 \%$ who actually reported that information in the works reported. Almost $40 \%$ of the works did not report any information on gender breakdown. Unfortunately for the age variable, $60 \%$ of the studies did not report findings considering the influence of age. Our work [22][25] indicated that these variables have an influence on olfactionbased mulsemedia QoE.

Finally, and importantly in terms of scents used in the olfaction-based mulsemedia literature, aspects to consider are: number of olfactory components used; scent types inclusive of the balance in terms of using pleasant scents only, unpleasant scents only and a mix of pleasant and unpleasant scent types. ISO 5496:2006 standard [64] states that no more than 10 scents should be used in subjective evaluation of olfaction. $92 \%$ of the experiments reported complied with this recommendation. Of more concern is the breakdown of scents used that have employed pleasant scents only. With $77 \%$ of odors used as being pleasant, we assume the reason for this was that using pleasant scents would be more enjoyable and result in higher QoE. Olfaction-based mulsemedia quality evaluations should, however, include scent types that are both pleasant and unpleasant scent types.

To conclude, we have highlighted a diverse range of variables which should be considered when performing olfaction-based mulsemedia evaluations. This section has shown a fragmented approach in some aspects whilst commonality in others. The next section reports the laboratory environments, assessment methodology, olfactionbased mulsemedia presentation equipment, screening approach, questionnaires, timings, video and scents, and assessors.

\section{MULTISENSORIAL EXPERIENCE BASED RECOMMENDATIONS FOR EXECUTION OF OLFACTION-BASED MULSEMEDIA QUALITY EVALUATION}

Considering the approaches and discussions presented in the literature and based on the authors own experiences, it is clear that the olfaction-based mulsemedia community has used diverse means to perform QoE evaluation. A commonality in terms of approach is required going forward. In this context, we suggest, based on our collective experiences of performing olfaction-based mulsemedia quality evaluation and expertise from the community, a number of recommendations for the execution of subjective testing involving olfaction. They recommendations are presented in an easy to understand manner with the motivation that the novice and experienced researcher alike can benefit from their presentation. We classify these recommendations into the following sub-categories:

(1) Assessor screening and training;

(2) Olfaction-based mulsemedia equipment, and

(3) Laboratory design and experimental design and methodology

\subsection{Assessor screening and training}

(a) In order to be eligible, assessors should not be involved in any sensory analysis in the twenty four hours preceding the tests.

Justification: This recommendation is based on the requirement to have "contamination" free reporting of user experiences. Considering olfaction in 
particular, assessor fatigue in the form of olfactory adaptation can severely influence potential user ratings of olfaction-based mulsemedia QoE.

(b) In order not to be affected by result contamination, assessors should not be affected by cold and flu, have good dental and overall body hygiene.

Justification: The ability to perceive an olfactory component unhindered by any degradation of the olfactory capability is crucial to, as per justification a.a, provide contamination free results. The variable perception as is, is complex enough, without additional variables influencing the user perception.

(c) Assessors should not have used any perfume, deodorants or aftershave before testing.

Justification: The presence of other factors, which also have associated an olfactory aspect should be minimized. These can severely influence the results of any tests in relation to both the subject wearing the additional scents and subsequent test participants.

(d) Assessors should avoid eating food, drinking tea/coffee or chewing gum at least two hours before any testing.

Justification: Since a well reported relationship (i.e. we can smell via taste stimulations [85] exists between the olfactory and gustatory senses, the rationale for this recommendation is the minimization of the presence of any factors which could influence user olfactory perception.

(e) Assessors should not be pregnant.

Justification: It is reported in the literature that a correlation exists between pregnancy and a person's perception of olfactory stimuli [85]. This is in order to ensure unbiased olfactory perception, but additionally to ensure safety of both mother and baby by preventing them from being exposed to odour substances.

(f) Assessors should not be forced to participate in the study (should be willing to take part).

Justification: Irrespective of whether an assessment is to measure user QoE of audio, visual, tactile, olfactory or gustatory experiences, it is crucial that assessors are providing unbiased results. In this context, a genuine interest and willingness to partake in the assessment is key aspect of screening assessors.

(g) Assessors should be healthy, especially free from allergies.

Justification: Since olfaction is a chemical media and with a motivation to ensure safety of all test subjects, assessors should provide informed consent which verifies that they are free from allergies which may cause any allergic reactions to the olfactory stimuli.

(h) Assessors should be screened for anosmia.

Justification: For useful result collection, the assessors should be screened to ensure they are capable of detecting the olfactory components that will be used as part of the assessment. The main motivation here is to provide useful results. The ISO standard 5496 [76] provides guidelines on how to perform such screening.

(i) Assessors should be screened based on visual capability according to existing standards such as [67]. 
Justification: Since olfaction-based mulsemedia is based on the integration of the olfaction and multimedia components, assessors should be screened as per appropriate standards from the traditional domain. This is as the literature reports that the visual and olfactory senses can significantly influence other senses, very important in particular given the synaesthesia phenomenon as outlined in detail in [84].

(j) A process involving test assessor familiarization and training with concern to the detection of odors should take place before undertaking the actual tests.

Justification: Because of the "novelty" of olfaction (or other multi-sensory multimedia), a detailed training phase should be considered. This is based on observations during pilot testing whereby initially assessors were tolerant of a wide range of "errors" simply to experience olfaction-enhanced multimedia and became more sensitive on repetition. Training sequences and odors should comprise of odoriferous substances representative of several groups of odor (pleasant/unpleasant) as well as substances that the assessors will evaluate during the actual evaluations.

\subsection{Olfaction-based mulsemedia equipment and laboratory design}

(k) Unique to olfaction, a mechanism to remove lingering scents is required after each test sequence.

Justification: The continued presence (lingering) of an olfactory component in the vicinity of an assessor's olfactory field can adversely affect or indeed enhance users' QoE. A controlled environment which supports a method to extract unwanted olfactory components outside of the particular time sequencing is necessary to ensure consistency across different assessors in terms of the presentation.

(l) Odors should be protected from light, and stored in sealable bags in a cool place (approx +5 degrees Celsius).

Justification: Storage within these types of conditions is to ensure consistency of the olfactory component (as much as possible) in terms of intensity. As was outlined in the research challenges section of this work, it is likely that varying intensity levels of the olfactory component has an effect on user QoE.

(m)The tests should be performed in an environment with minimal assessor distraction [54]

Justification: To ensure repeatable research, contamination-free assessor environments and unbiased results, accepted ISO standards like [62] should be employed. For example, it is recommended that the walls in the rooms where olfactory evaluations are performed should be matt-off-white [62]. The purpose for this is to minimize the effects of phenomena like synesthesia.

(n) Facilitate adjustable seating if possible to ensure assessor comfort.

Justification: Since the primary motivation of olfaction-based mulsemedia QoE, assessor comfort is crucial as are other infrastructure factors in the evaluation room such as temperature etc. Also key to unbiased olfactory QoE results between assessors is consistency between assessors olfactory fields so that assessors do not "miss out" on the olfactory component due to height, posture etc. In addition, consistency between assessors in terms of their distance from the 
olfaction-based mulsemedia presentation system to ensure equal delivery times, is an important consideration.

\subsection{Experimental design and methodology}

(o) Test breaks should be used to avoid assessor fatigue in terms of olfactory adaptation. Hence, our recommendation is that in any tests that extend 30 minutes, assessors are given a break of 15-20 minutes upon 30 minutes post-test start time.

Justification: Olfactory adaptation can result in assessors not perceiving the presence of olfactory stimuli. It is salient to conclude that when assessing olfaction-based mulsemedia QoE, this would have devastating consequences for the consistency of findings.

(p) The assessors should be exposed to no more than 10 odoriferous substances per session in order to avoid olfactory adaption. The over exposure to olfactory receptors of olfactory components can results in assessors being unable to perceive the scents.

(q) There should be an explanation to the assessors of the methodology that is employed.

Justification: This recommendation is omnipresent in many audiovisual assessment methodologies. Assessors should be directed to base their judgment on their overall experience in terms of the wordings of the subjective scales used.

(r) The use of interview questions to complement Likert scale type data capture is recommended to ensure maximum information from assessors on their perception of the olfaction based mulsemedia experiences is achieved.

Justification: Borrowing recommendations on approaches from the psychophysical fields, structured and controlled interview questions can support new learning and findings and add to our understanding of olfaction-enhanced mulsemedia QoE.

\subsection{QoE questionaires and analysis}

(s) A plain language test description document is recommended to be distributed to the assessors in order to help them understand the goal of the tests and provide answers in that context.

Justification: Different test goals significantly influence the content of the testing questionnaires. There are diverse avenues in terms of QoE assessment which can focus on studying the influence on user overall satisfaction, their learning outcome, the effect of quality of delivery, the influence of environment, etc. Not all of them are compulsory.

(t) Test questions could capture the effects of quality of delivery which are known to severely influence the user perception of quality of individual mulsemedia components such as: blockiness and blurness (for video), pre-echo (for audio), detection of stimulus (for olfaction).

Justification: User QoE is difficult to be directly measured and therefore diverse other factors which influence user QoE levels, but are easier assessed are used instead. 
(u) Test questions could assess the effect of factors influencing the user perception of quality of combined multisensory content such as: synchronization and masking. Justification: User QoE is very much affected by lack of synchornisation between different sensorial content and this happens easily during mulsemedia content distribution, especially via diverse network types.

(v) Test questions could capture factors influencing the user perception of quality in terms of contextual fit such as: sense of reality.

Justification: User QoE includes a component highly dependent on the user perceived sense of reality, which is even stronger enhanced in mulsemedia context.

(w) Test questions could focus on assessing the effect of factors influencing the user sense of satisfaction.

Justification: Overall user QoE is influenced by user sense of satisfaction or enjoyment, which is an important factor to be considered in any perceptual testing.

(x) Test questions could analyze the effect of mulsemedia content on performing useful tasks such as learning.

Justification: Learning outcome, problem completion time or rate and task efficiency are some important metrics to be considered when any mulsemediaenhanced content is delivered as part on an enhanced learning process, innovative problem solving stage or novel task completion exercise.

\section{CONCLUSION}

The recent addition of so called new media components to the traditional multimedia content has been very well received and an increasing number of users are accessing multisensorial media (mulsemedia). Capturing mulsemedia user perceived QoE is non-trivial mostly due to the number and various types of media components which are presented in synchronised manner. As there are no standardized methodologies to conduct subjective mulsemedia quality assessment, researchers have used different approaches to assess user QoE of mulsemedia applications. This paper focused on olfactory-based mulsemedia applications, and presented a review of QoE assessment solutions employed in the latest reported research works in this space. The comparative discussion considered methodologies, rating scales, test sample sizes and balance, assessor screening and training, number and type of scents used and laboratory environment. Then, the article provided a tutorial on the methodologies employed by the authors in their own research, considering the same aspects, which are highly relevant for QoE assessment. Finally, as one of the most important contributions of this paper, this paper presents a set of recommendations for mulsemedia quality evaluation based on author experience in the area of olfaction-based mulsemedia.

\section{ACKNOWLEDGEMENT}

G. Ghinea and G.-M. Muntean would like to acknowledge the support of European Union's Horizon 2020 Research and Innovation programme under Grant Agreement no. 688503 for the research performed in the NEWTON project (http://www.newtonproject.eu). 


\section{REFERENCES}

[1] N. Murray, Y. Qiao, B. Lee, AK Karunakar, G.-M. Muntean, "Subjective Evaluation of Olfactory and Visual Media Synchronization” In Proceedings of ACM Multimedia Systems conference, pp. 162-171, Feb 26 - March 1, Oslo, Norway. 2013.

[2] F. Danieau, A. Lecuyer, P. Guillotel, J. Fleureau, N. Mollet and M. Christie, "Enhancing Audiovisual Experience with Haptic Feedback: A Survey on HAV”. In IEEE Transactions on Haptics, vol. 6, issue 2, pp 193-205, 2012.

[3] T. Narumi, S. Nishizaka, T. Kajinami, T. Tanikawa and M. Hirose, "Augmented Reality Flavors: Gustatory Display Based on Edible Marker and Cross-Modal Interaction". In Proceedings of the ACM CHI Conference on Human Factors in Computing Systems., pp. 93-102, 2011.

[4] G. Ghinea, S. R. Gulliver and F. Andres (eds.), "Multisensorial Media Advances and Applications: New Developments in mulsemedia”, IGI Global, 2011.

[5] G. Ghinea, C. Timmerer, W. Lin and S. R. Gulliver, "mulsemedia: State of the Art, Perspectives, and Challenges". ACM Trans. Multimedia Comput. Commun. Appl. 11, 1s, Article 17 (October 2014), 23 pages. DOI=10.1145/2617994 http://doi.acm.org/10.1145/2617994

[6] P. Le Callet, S. Möller and A. Perkis, Qualinet White Paper on "Definitions of Quality of Experience". European Network on Quality of Experience in Multimedia Systems and Services (COST Action IC 1003), Lausanne, Switzerland, Version 1.1, June 3, 2012.

[7] LA Rowe and R. Jain, "ACM SIGMM retreat report on future directions in multimedia research" In ACM Transactions on Multimedia Computing, Communications, and Applications (TOMM), Volume 1 Issue 1, Pages 3-13, 2005.

[8] Z. Yuan, S. Chen, G. Ghinea and G.-M. Muntean, "Beyond Multimedia Adaptation: Quality of Experience-aware Multi-sensorial Media Delivery". In IEEE Trans. on Multimedia, vol. 17, issue 1, pp.104-117, 2015.

[9] M. Seufert, S. Egger, M. Slanina, T. Zinner, T. Hossfeld, P. Tran-Gia, "A Survey on Quality of Experience of HTTP Adaptive Streaming”, IEEE Communications Surveys \& Tutorials, vol. 17, no. 1, pp. 469-492, 2015

[10] D. S. Nunes, P. Zhang, J. S. Silva, "A survey on human-in-the-loop applications towards an internet of all”, IEEE Communications Surveys \& Tutorials, vol. 17, no. 2, pp. 944-965, 2015

[11] P. Juluri, V. Tamarapalli, D. Medhi, "Measurement of Quality of Experience of Video-on-Demand Services: A Survey”, IEEE Communications Surveys and Tutorials, vol. 18, no. 1, pp. 401-418, 2016

[12] P. Seeling, M. Reisslein, "Video Transport Evaluation with H.264 Video Traces", IEEE Communications Surveys and Tutorials, vol. 14, no. 4, pp. $1142-1165,2012$

[13] M. Kennedy, A. Ksentini, Y. Hadjadj-Aoul, G.-M. Muntean, "Adaptive Energy Optimization in Multimedia-centric Wireless Devices: A Survey", IEEE Communications Surveys and Tutorials, vol. 15 , No. 2, , pp. 768-786, 2013

[14] Y. Chen, K. Wu, Q. Zhang, "From QoS to QoE: A Tutorial on Video Quality Assessment", IEEE Communications Surveys and Tutorials, vol. 17, no. 2, pp. 1126-1165, 2015

[15] M. Obrist, A.N. Tuch and K. Hornbaek, "Oppurtunities for odor: experiences with smell and implications for technology". In Proceedings of SIGCHI Conference on Human Factors in Computing Systems, pp 2843-2852, 2014.

[16] G. Ghinea and O. A. Ademoye, "Olfaction-enhanced multimedia: perspectives and challenges". In Multimedia Tools and Applications, vol. 55, no. 3, pp. 601-626, 2011.

[17] N. Murray, B. Lee, Y. Qiao and G.-M. Muntean, "Olfaction enhanced Multimedia: A survey of application domains, Displays and Research Challenges”. In ACM Computing Surveys, Vol. 48, Issue 4, Article 56, May 2016.

[18] Y. Yanagida, "A survey of olfactory displays: Making and delivering scents". In IEEE Sensors, pp. 1-4, 2012.

[19] ISO/IEC FDIS 23005 Information Technology - Media context and control- Part 1-7, ISO Publication (2010)

[20] B. Rainer, C. Timmerer and M. Waltl, "Recommendations for the subjective evaluation of sensory experience”, In: Fourth International Workshop on Perceptual Quality of Systems, 2013.

[21] ITU-T P.910. Subjective video quality assessment methods for multimedia applications, 2008. 
[22] A. Hamam, M. Eid, A. El Saddik, and N. D. Georganas, "A fuzzy logic system for evaluating quality of experience of haptic-based applications. In International Conference on Human Haptic Sensing and Touch Enabled Computer Applications", Springer Berlin Heidelberg, June 2008

[23] A. Hamam, M. Eid, A. El Saddik, and N. D. Georganas, "A quality of experience model for haptic user interfaces." Ambi-Sys workshop on Haptic user interfaces in ambient media systems, Institute for Computer Sciences, Social-Informatics and Telecommunications Engineering, February 2008

[24] N. Murray, Y. Qiao, B. Lee, A K Karunakar, G.-M. Muntean, "Age and Gender Influence on Perceived Olfactory and Visual Media Synchronization". In IEEE International Conference on Multimedia and Expo (ICME), pp. 1-6, July 15 - 19, San Jose, California, USA, 2013.

[25] N. Murray, Y. Qiao, B. Lee, and G.-M. Muntean, "User Profile Based Perceived Olfactory and Visual Media Synchronization". In ACM Transactions on Multimedia Computing, Communications, and Applications (TOMM), vol. 11, no. 1, article 11, 2014.

[26] N. Murray, B. Lee, Y. Qiao and G.-M. Muntean, "Multiple-Scent enhanced multimedia synchronization". In ACM Transactions on Multimedia Computing Communications and Applications (TOMM), Special Issue on Mulsemedia, vol. 10, no. 1, article no. 12, 2014.

[27] C. Timmerer, M. Waltl, B. Rainer and N. Murray, "Sensory Experience: Quality of Experience Beyond Audio-Visual", in "Quality of Experience: Advanced Concepts, Applications and Methods" (S. Mauller and A. Raake, eds.), Springer, Heidelberg, Germany, pp. 351-365, 2014

[28] N. Murray, B. Lee, Y. Qiao and G.-M. Muntean, "Impact of Scent type on Olfaction enhanced Multimedia Quality of Experience". In IEEE Transactions on Systems, Man, and Cybernetics: Systems vol. PP, no. 99, 2016, http://ieeexplore.ieee.org/document/7457332/

[29] O. A. Ademoye and G. Ghinea, "Synchronization of Olfaction-enhanced Multimedia". In IEEE Transactions on Multimedia, vol. 11 no. 3, pp. 561-565, 2009.

[30] O. A. Ademoye and G. Ghinea, "Information recall task impact in olfaction-enhanced multimedia". In ACM Transactions on Multimedia Computing, Communications, and Applications (TOMM), vol. 9, issue 3, article 17, 2013.

[31] G. Ghinea and O. A. Ademoye, "The Sweet Smell of Success: Enhancing Multimedia Applications with Olfaction", In ACM Transactions on Multimedia Computing, Communications and Applications (TOMM), vol. 8, issue 1, article 2, 2012.

[32] Exhalia website, http://www.exhalia.com - accessed on 14.01.2016

[33] G. Ghinea and O. A. Ademoye, "Perceived Synchronization of Olfactory Multimedia". In IEEE Transactions on Systems, MAN, and Cybernetics-Part A: Systems and Humans, vol. 40, no. 4, pp 657-663, 2010.

[34] G. Ghinea, O.A. Ademoye, "Olfaction-enhanced multimedia: Bad for information recall?" In International conference on Multimedia and Expo (ICME), pp. 970-973, 2009.

[35] G. Ghinea and O.A. Ademoye, "User perception of media content association in olfaction-enhanced multimedia". In ACM Transactions on Multimedia Computing, Communications, and Applications (TOMM), vol. 8, no. 4, article 52, 2012.

[36] F. Anderson, M. Annett, W.F. Bischof and P. Boulanger, "Virtual Equine Assisted Therapy". In IEEE Virtual Reality Conference, pp.255-256, 2010.

[37] E. Arroyo, T. Selker, and A. Stouffs, "Interruptions as multimodal outputs: Which are less disruptive?" In IEEE International Conference on Multimodal Interfaces, pp. 479-482, 2002.

[38] B. Berglund, U. Berglund, T. Engen, and T. Lindvall, "The effect of adaptation on odor detection". In Perception \& Psychophysics, vol 9, issue 5, pp 435-438, 1971..

[39] ITU-T BT.500. Methodology for the subjective assessment of the quality of television pictures, 2002

[40] A. Bodnar, "AROMA: Ambient awareness through Olfaction in a Messaging Application". In Proc. 6th International Conference on Multimodal Interfaces (ICMI), pp. 183-190, 2004..

[41] S. A. Brewster, D.K. McGoookin and C.A. Miller, "Olfoto: designing a smell-based interaction". In ACM SIGCHI Conference on Human Factors in Computing Systems, pp. 653-662, 2006..

[42] Daleir Website, www.daleair.com - accessed on 14.01.2016

[43] B. R. Brkic, A. Chalmers, K. Boulanger, S. Pattanaik, J. Covington, "Cross-modal effects of smell on the real-time rendering of grass”. In 25th Spring Conference on Computer Graphics (SCCG), pp. 161$166,2009$.

[44] J.-C. Chebat and R. Michon, "Impact of ambient odors on mall shoppers' emotions, cognition, and spending: A test of competitive causal theories". In Journal of Business Research, vol. 56, issue 7, pp. 529-539, 2003. 
[45] R. Chi-Wai Kwok, S. Han Cheng, H. Ho-Shing Ip and J. Siu-Lung Kong, "Design of affectively evocative smart ambient media for learning". In Computers and Education, vol. 56, no. 1, pp. 101-111, 2011.

[46] H.O. Dinh, N. Walker, C. Song, A. Kobayashi and L.F. Hodges, "Evaluating the importance of MultiSensory Input on Memory and the Sense of Presence in Virtual Environments". In IEEE Virtual Reality, pp. 222-228, 1999.

[47] M. A. Garcia-Ruiz, A. Edwards, R. Aquino-Santos, O. Alvarez-Cardenas and M.G. Mayoral-Baldivia, "Integrating the Sense of Smell in Virtual Reality for Second Language Learning". World Conference on E-Learning in Corporate, Government, Healthcare, and Higher Education, pp. 2647-2652, 2008.

[48] P. Huang, Y. Ishibashi, N. Fukushima and S. Sugawara, "Effect of Dynamic Control of Fragrance Output Timing in Networked Virtual Environment". In 18th Asia-Pacific Conference on Communications, pp. 104-109, 2012.

[49] S. Hoshino, Y. Ishibashi, N. Fukushima, and S. Sugawara, "QoE Assessment in Olfactory and Haptic Media Transmission: Influence of Inter-Stream Synchronization Error". In IEEE Int. Workshop Technical Committee on Communications Quality and Reliability, pp 1-6, 2011.

[50] H. Matsukura, T. Nihei and H. Ishida, "Multi-sensorial field display: Presenting spatial distribution of airflow and odor". IEEE Virtual Reality Conference (VR), Singapore, pp. 119-222, 2011.

[51] T. Narumi, T. Kajinami, S. Nishizaka, T. Tankiawa and M. Hirose, "Pseudo-Gustatory Display System based on Cross-Modal Integration of Vision, Olfaction and Gustation". In IEEE Virtual Reality, pp. 127-130, 2011.

[52] D. Noguchi, S. Sugimoto, Y. Bannai and K. Okada, "Time Characteristics of Olfaction in a Single Breath", ACM CHI Conference on Human Factors in Computing Systems (CHI2011), pp. 83-92, 2011.

[53] D. Noguchi, K. Ohtsu, Y. Bannai, and K. Okada, "Scent Presentation Expressing Two Smells of Different Intensity Simultaneously". In Joint Virtual Eurographics Conference on Virtual Environments, pp. 53-60, 2008.

[54] K. Tomono, H. Katsuyama and A. Tomono, "Scent Emitting Display Panel and Its Psychological effects". In Journal of Information and Media Technologies, vol. 7, issue. 2, pp.761-769, 2012.

[55] R. Tortell, D.P. Luigi, A. Dozois, S. Bouchard, J.F. Morie and D. Ilan "The effects of scent and game play experience on memory of a virtual environment". In IEEE Virtual Reality, vol. 11, no. 1, pg. 6168, 2007.

[56] Anthrotronix website, http://www.anthrotronix.com/our-work/simulation-and-training/

[57] J.S. Warm, W.N. Dember and E. Parasuraman,"Effects of Olfactory Stimulation on Performance and Stress in a Visual Sustained Attention Task". In Journal of the Society of Cosmetic Chemists, vol 42, pp. 199-210, 1991.

[58] D. Warnock, "A subjective evaluation of multimodal notifications". In 5th International Conference on Pervasive Computing Technologies for Healthcare (PervasiveHealth), pp 461-468, 2011.

[59] A. Yazdani, E. Kroupi, J. Vesin and T. Ebrahimi, "Electroencephalogram alterations during perception of pleasant and unpleasant odors", In 4th International Workshop on Quality of Multimedia Experience (QoMEX), pp. 272-277, 2012.

[60] Z. Huang and K. Nahrstedt, "Perception-based playout scheduling for high-quality real-time interactive multimedia". In Proceedings of IEEE International Conf. on Computer Communications (INFOCOM) pp. 2786-2790, 2012.

[61] ITU-T P.913: Methods for the subjective assessment of video quality, audio quality and audiovisual quality of Internet video and distribution quality television in any environment, 2014.

[62] ISO standard (ISO/IEC 8589:2007), "Sensory analysis - General guidance for the design of test rooms", 2007.

[63] R.E. Cytowic.. Synesthesia: A Union of the Senses (2nd edition), The MIT Press. Cambridge, MA, 2002 .ISBN 0-262-03296-1.

[64] ISO 5496:2006. Sensory analysis - Methodology - Initiation and training of assessors in the detection and recognition of odors, 2006.

[65] Z. Yuan, S. Chen, G. Ghinea and GM. Muntean, "User quality of experience of mulsemedia applications", In ACM Transactions on Multimedia Computing, Communications, and Applications (TOMM), vol. 11, no. 1, article 15, 2014 
[66] ITU-T Recommendation P.911, "Subjective Audiovisual Quality Assessment Methods for Multimedia Applications," Dec. 1998

[67] ITU-T Recommendation P.913, "Methods for the Subjective Assessment of Video Quality, Audio Quality and Audiovisual Quality of Internet Video and Distribution Quality Television in Any Environment", Int. Telecommunications Union, Geneva, Switzerland, Jan. 2014

[68] A. Nambu, T. Narumi, K. Nishimura, T. Tanikawa, M. Hirose, "Visual-Olfactory display using Olfactory Sensory Map", In proceedings of IEEE Virtual Reality Conference, pp. 39-42, 2010.

[69] B. Ramic, A. Chalmers, J. Hasic, S. Rizvic, "Selective Rendering in a multimodal environment: Scent and graphics", In Proceedings of the 23rd Spring Conference on Computer Graphics, pp. 147-151, 2007.

[70] T. Tanikawa, M. Hirose, "A Study of Multi-modal Display System with Visual Feedback", In International Symposium on Universal Communication, pp. 285-292, 2008.

[71] T. Sikora, "The MPEG-7 visual standard for content description: An overview," IEEE Trans. Circuits Syst. Video Technol., vol. 11, no. 6, pp. 696-702, Jun. 2001.

[72] N. Murray, B. Lee, Y. Qiao, G.-M. Muntean, "The influence of human factors on olfaction based mulsemedia quality of experience", In Special Session on User Factors in Multimedia Experiences at the 8th International Conference on Quality of Multimedia Experience (QoMEX 2016) June 2016, in Lisbon, Portugal, 2016.

[73] G.-M. Muntean, "Efficient Delivery of Multimedia Streams over Broadband Networks using QOAS", IEEE Trans. on Broadcasting, vol. 52, no. 2, June 2006, pp. 230-235

[74] G.-M. Muntean, P. Perry, L. Murphy, "Objective and Subjective Evaluation of QOAS Video Streaming over Broadband Networks", IEEE Trans. on Network and Service Management, vol. 2, no. 1, 2005, pp. 19-28

[75] Z. Yuan, G.-M. Muntean, "A Prioritized Adaptive Scheme for Multimedia Services over IEEE 802.11 WLANs", IEEE Trans. on Network and Service Management, vol. 10, no. 4, Dec. 2013, pp. 340-355

[76] ISO Standard 5496, "Sensory Analysis - Methodology - Initiation and training of assessors in the detection and recognition of odours" 2006

[77] ISO/IEC FDIS 23005-2 Information Technology - Media context and control- Part 2: Control Information, ISO Publication (2010)

[78] ISO/IEC FDIS 23005-3 Information Technology - Media context and control- Part 3: Sensory Information, ISO Publication (2010)

[79] ISO/IEC FDIS 23005-4 Information Technology - Media context and control- Part 4: Virtual World Object Characteristics, ISO Publication (2010)

[80] ISO/IEC FDIS 23005-5 Information Technology - Media context and control- Part 5: Data formats for Interaction Devices, ISO Publication (2010)

[81] ISO/IEC FDIS 23005-6 Information Technology - Media context and control- Part 6: Common Types and Tools, ISO Publication (2010)

[82] ISO/IEC FDIS 23005-7 Information Technology - Media context and control- Part 7: Reference Software and Conformance, ISO Publication (2010)

[83] P. T. Kovács, N. Murray, G. Rozinaj, Y. Sulema, "Application of Immersive Technologies for Education: State Of The Art" In 9th International Conference on Interactive Mobile Communication Technologies and Learning, IMCL2015, Special Session on Immersive Technologies for Effective Learning, 2015

[84] R. E. Cytowic. 2002. Synesthesia: A Union of the Senses (2nd edition), The MIT Press. Cambridge, MA .ISBN 0-262-03296-1.

[85] G. Calvert, C. Spence, B.E. Stein. 2004. The Handbook of Multisensory Processes. Massachusetts Institute of Technology Press, ISBN: 9780262033213.

[86] A. N. Gilbert and C. J. Wysocki. Quantitative assessment of olfactory experience during pregnancy. Adv Psychosom Med vol. 53, pp. 693-700, 1991

[87] M. Obrist, E. Gatti, E. Maggioni, CT Vi, C. Velasco. "Multisensory Experiences in HCI". In IEEE Transactions on Multimedia, Vol. 24, Issue 2, pp. 9-13, June 2017.

[88] N. S. Herrera and R. P. McMahan, "Development of a Simple and Low-Cost Olfactory Display for Immersive Media Experiences," in Proceedings of the 2nd ACM International Workshop on Immersive Media Experiences - ImmersiveMe '14, 2014, pp. 1-6. 
[89] Y. Yanagida, S. Kawato, H. Noma, A. Tomono, and N. Tesutani, "Projection based olfactory display with nose tracking," in IEEE Virtual Reality 2004, pp. 43-50.

[90] D. McGookin and D. Escobar, "Hajukone," in Proceedings of the 2016 CHI Conference Extended Abstracts on Human Factors in Computing Systems - CHI EA '16, 2016, pp. 1721-1728.

[91] B. Ramic-Brkic and A. Chalmers, "Olfactory Adaptation in Virtual Environments," ACM Trans. Appl. Percept., vol. 11, no. 2, pp. 1-16, Jun. 2014.

[92] J. N. Kaye, "Symbolic Olfactory Display," Massachusetts Institute of Technology, 2001.

[93] N. Ranasinghe, G. Suthokumar, K.-Y. Lee, and E. Y.-L. Do, "Digital Flavor," in Proceedings of the 2015 ACM on International Conference on Multimodal Interaction - ICMI '15, 2015, pp. 139-146.

[94] S. A. Seah, D. Martinez Plasencia, P. D. Bennett, A. Karnik, V. S. Otrocol, J. Knibbe, A. Cockburn, and S. Subramanian, "SensaBubble," in Proceedings of the 32nd annual ACM conference on Human factors in computing systems - CHI'14, 2014, pp. 2863-2872.

[95] E. B. Saleme and C. A. S. Santos, "Playsem: A platform for rendering mulsemedia compatible with mpeg-v," in Proceedings of the 21st Brazilian Symposium on Multimedia and the Web, WebMedia'15, 145-148, 2015.

[96] M. Waltl B. Rainer, C. Timmerer, and H. Hellwagner, "An end-to-end tool chain for sensory experience based on MPEG-V," Image Communication., 28(3), 136-150, 2013.

[97] N. Diolaiti, G. Niemeyer, F. Barbagli, and J.K. Salisbury, (2006). Stability of haptic rendering: Discretization, quantization, time delay, and coulomb effects. IEEE Transactions on Robotics, 22(2), 256-268

[98] M. Rank, Z. Shi, and S. Hirche. Perception of delay in haptic telepresence systems. Presence, 19(5), 389-399, 2010.

[99] Z. Yuan, T. Bi, G.M. Muntean, and G. Ghinea, G. Perceived synchronization of mulsemedia services. IEEE Transactions on Multimedia, 17(7), 957-966, 2015.

[100] O.A. Ademoye, N. Murray, G.M. Muntean, and G. Ghinea, Audio Masking Effect on InterComponent Skews in Olfaction-Enhanced Multimedia Presentations. ACM Transactions on Multimedia Computing, Communications, and Applications (TOMM), 12(4), 51, 2016.

[101] J. M. Silva, M. Orozco, J. Cha, A. E. Saddik, and E. M. Petriu, "Human perception of haptic-tovideo and haptic-to-audio skew in multimedia applications," ACM Trans. Multimed. Comput. Commun. Appl., 9(2), pp. 1-16, 2013.

[102] A. Tatematsu, Y. Ishibashi, N. Fukushima, and S. Sugawara, "QoE assessment in haptic media, sound and video transmission: Influences of network latency," in 2010 IEEE International Workshop Technical Committee on Communications Quality and Reliability (CQR 2010), 1-6, 2010.

[103] Q. Zeng, Y. Ishibashi, N. Fukushima, S. Sugawara, and K. E. Psannis, "Influences of interstream synchronization errors among haptic media, sound, and video on quality of experience in networked ensemble," in 2013 IEEE 2nd Global Conference on Consumer Electronics (GCCE), 466470, 2013.

[104] N. Ranasinghe, A. Cheok, R. Nakatsu, and E. Y. L. Do. Simulating the sensation of taste for immersive experiences. In Proceedings of the 2013 ACM international workshop on Immersive media experiences, 29-34, 2013

[105] D. Dmitrenko, C. T. Vi, M. Obrist, "A Comparison of Scent-Delivery Devices and Their Meaningful Use for In-Car Olfactory Interaction” In Proceedings of the 8th International Conference on Automotive User Interfaces and Interactive Vehicular Applications, pp. 23-26 